\title{
Ballistocardiography and Seismocardiography: A Review of Recent Advances
}

\author{
Omer T. Inan, Member, IEEE, Pierre-Francois Migeotte, Member, IEEE, Kwang-Suk Park, Senior Member, IEEE, \\ Mozziyar Etemadi, Student Member, IEEE, Kouhyar Tavakolian, Member, IEEE, Ramon Casanella, Member, IEEE, \\ John Zanetti, Jens Tank, Irina Funtova, G. Kim Prisk, Senior Member, IEEE, and Marco Di Rienzo, Member, IEEE
}

Abstract-In the past decade, there has been a resurgence in the field of unobtrusive cardiomechanical assessment, through advancing methods for measuring and interpreting ballistocardiogram (BCG) and seismocardiogram (SCG) signals. Novel instrumentation solutions have enabled BCG and SCG measurement outside of clinical settings, in the home, in the field, and even in microgravity. Customized signal processing algorithms have led to reduced measurement noise, clinically relevant feature extraction, and signal modeling. Finally, human subjects physiology studies have been conducted using these novel instruments and signal processing tools with promising clinically relevant results. This paper reviews the recent advances in these areas of modern BCG and SCG research.

Index Terms-Ballistocardiogram (BCG), cardiomechanical signals, noninvasive physiologic monitoring, seismocardiogram (SCG), ubiquitous health.
Manuscript received May 14, 2014; revised August 20, 2014; accepted September 29, 2014. Date of publication; date of current version. The work of P. F. Migeotte was supported by the Belgian Federal Science Policy Office via the European Space Agency PRODEX program (ILSRA-2009-0729). The work of G. K. Prisk was supported by the National Space Biomedical Research Institute through NASA NCC9-58. The work of J. Tank and I. I. Funtova were supported by the German Space Agency (DLR) under Grant 50WB1117. The work of M. Di Rienzo was supported in part by the Italian Space Agency through ASI 2013-061-I.0 and ASI 2013-079-R.0.

O. T. Inan is with the School of Electrical and Computer Engineering, Georgia Institute of Technology, Atlanta, GA 30308 USA (e-mail: oeinan@ gmail.com).

P.-F. Migeotte is with the Department of Cardiology, Universite Libre de Bruxelles 1050, Brussels, Belgium (e-mail: Pierre-Francois.Migeotte@ulb.ac.be).

K.-S. Park is with the Department of Biomedical Engineering, Seoul National University, Seoul 110-799, Korea (e-mail: kspark@ bmsil.snu.ac.kr).

M. Etemadi is with the Department of Bioengineering and Therapeutic Sciences, University of California at San Francisco, San Francisco, CA 94143 USA (e-mail: mozziyar.etemadi@ucsf.edu).

$\mathrm{K}$. Tavakolian is with the Department of Electrical Engineering, University of North Dakota, Grand Forks, ND 58202 USA (e-mail: kouhyart@ gmail.com).

R. Casanella is with the Instrumentation, Sensors, and Interfaces Group, Universitat Politecnica de Catalunya, 08034 Barcelona, Spain (e-mail: ramon. casanella@upc.edu).

J. Zanetti is with Acceleron Medical Systems, Arkansaw, WI 54721 USA (e-mail: jmzsenior@gmail.com).

J. Tank is with the Klinsche Pharmakologie, Medizinische Hochschule Hannover, 30625 Hannover, Germany (e-mail: Tank.Jens@mh-hannover.de).

I. Funtova is with the Laboratory for Autonomic Regulation of Cardiorespiratory System, Institute of Biomedical Problems, Russian Academy of Sciences, 123007 Moscow, Russian (e-mail: funtova.imbp@mail.ru).

G. K. Prisk is with the Department of Medicine and Radiology, University of California at San Diego, La Jolla, CA, 92093 USA (e-mail: kprisk@ucsd.edu).

M. Di Rienzo is with the Department of Biomedical Technology, Fondazione Don Carlo Gnocchi, ONLUS, 20133 Milano, Italy (e-mail: mdirienzo@ dongnocchi.it)

Color versions of one or more of the figures in this paper are available online at http://ieeexplore.ieee.org.

Digital Object Identifier 10.1109/JBHI.2014.2361732

\section{INTRODUCTION}

A $\mathrm{S}$ detailed in the following sections, the ballistocardiogram $(\mathrm{BCG})$ is a measurement of the recoil forces of the body in reaction to cardiac ejection of blood into the vasculature [1], while the seismocardiogram (SCG) represents the local vibrations of the chest wall in response to the heartbeat [2]. The BCG phenomenon was first observed in 1877 by Gordon, with the finding that, as a subject would stand on a weighing scale, the needle would vibrate synchronously to the subject's heartbeat [3]. Nearly 60 years later, Starr and colleagues created an instrument in the form of a table with a mobile top surface to measure the BCG in a repeatable scientific manner [1]. The SCG was first observed by Bozhenko in 1961, and was first applied in clinical studies 30 years later in 1991 by Salerno and Zanetti [4]. Throughout the 1900s, both BCG and SCG signals were heavily investigated and several publications appeared in major scientific and clinical journals (e.g., [4]-[7]). However, because of the advent of echocardiography and magnetic resonance imaging, and overly-cumbersome hardware, BCG and SCG were largely abandoned by the medical community [8].

Today, technological advancements largely simplify the measurement and assessment of these signals and open new perspectives in their clinical use. This paper reviews the instrumentation and signal processing advances which have helped to propel BCG and SCG into this revival. It also summarizes some of the key human subjects studies performed recently that support the use of BCG and SCG in extra-clinical applications.

\section{DESCRIPTION OF BCG AND SCG SignALS}

\section{A. BCG Signal Description}

At every heartbeat, the blood travelling along the vascular tree produces changes in the body center of mass. Body micromovements are then produced by the recoil forces to maintain the overall momentum. The BCG is the recording of these movements, can be measured as a displacement, velocity, or acceleration signal, and is known to include movements in all three axes. The longitudinal BCG is a measure of the head-to-foot deflections of the body, while the transverse BCG represents anteroposterior (or dorso-ventral) vibrations. The original bed- and table-based BCG systems focused on longitudinal BCG measurements, representing what was supposed to be the largest projection of the 3-D forces resulting from cardiac ejection [1]. Table I summarizes modern BCG measurement systems and their axes of measurement. Note that for some systems, head-to-foot and dorso-ventral forces are unavoidably, mixed 
TABLE I

MOdern BCG Systems AND THEIR CORRESPONDING MEASUREMENT AXES

\begin{tabular}{lll}
\hline \hline Modern BCG System & \multicolumn{1}{c}{ Axis } & \multicolumn{1}{c}{ Comments / Challenges } \\
\hline Accel. (0g) & All (3-D) & - Needs reduced gravity \\
Accel. (1g) & Head-to-foot & $\begin{array}{l}\text { - Placement affects signal shape } \\
\text { and amplitude }\end{array}$ \\
& & - Motion artifacts must be \\
& & detected and mitigated \\
Bed & Head-to-foot or & - Cross-axis coupling \\
& Dorso-ventral & - Changes in sleep position affect \\
& signal quality / shape \\
Chair & Head-to-foot or & - Posture affects signal quality \\
& Dorso-ventral & and repeatability \\
Weighing Scale & Head-to-foot & - Posture affects signal quality \\
& & and repeatability \\
& & - Motion artifacts must be \\
& & detected and mitigated \\
& & \\
\hline
\end{tabular}

together in the measurement, and this should be accounted for when interpreting results. However, in spite of the 3-D nature of the BCG, for a long period of time only the microdisplacements of the body along the longitudinal axis (head-to-foot) were considered. Currently, BCG is mainly measured using a force plate or force sensor placed on a weighing scale or under the seat of a chair, with the subject in a vertical position. Modern approaches to BCG measurement are discussed below in Section III.

It should be considered, however, that the gravity force and any contact of the body with external objects, including the floor and measuring devices, somewhat interferes with, or even impedes, the body displacement induced by the recoil forces. As a result, the BCG measurement on earth is always affected by some distortion. The ideal environment for assessing the BCG would be in microgravity settings, such as during space missions. Such experiments have been performed, and the results described below confirm that in microgravity the whole body recoil forces (BCG) are significant in all three dimensions [9]-[12]. Modeling studies examining the cardiogenic traction forces of the aorta have confirmed this finding as well [13].

\section{B. SCG Signal Description}

SCG is the measure of the thoracic vibrations produced by the heart's contraction and the ejection of blood from the ventricles into the vascular tree. Today, the SCG can readily be detected by placing a low-noise accelerometer on the chest. If a tri-axial accelerometer is used, SCG components are present in all three axes, each displaying a specific pattern [12], [14]. However, in the literature, the majority of studies on SCG only focus on the amplitude of the dorso-ventral component, although it is likely that additional biological information could be derived also from the analysis of the longitudinal and lateral SCG components, and from the analysis of the acceleration vector trajectory during the heart cycle. Unless the contrary is stated to be consistent with the prevalent literature only the dorso-ventral acceleration component of SCG will be considered in the remainder of this paper.

\section{BCG and SCG Waveforms}

For each heart contraction, a BCG and SCG waveform is generated. Each waveform is characterized by several peaks and val-

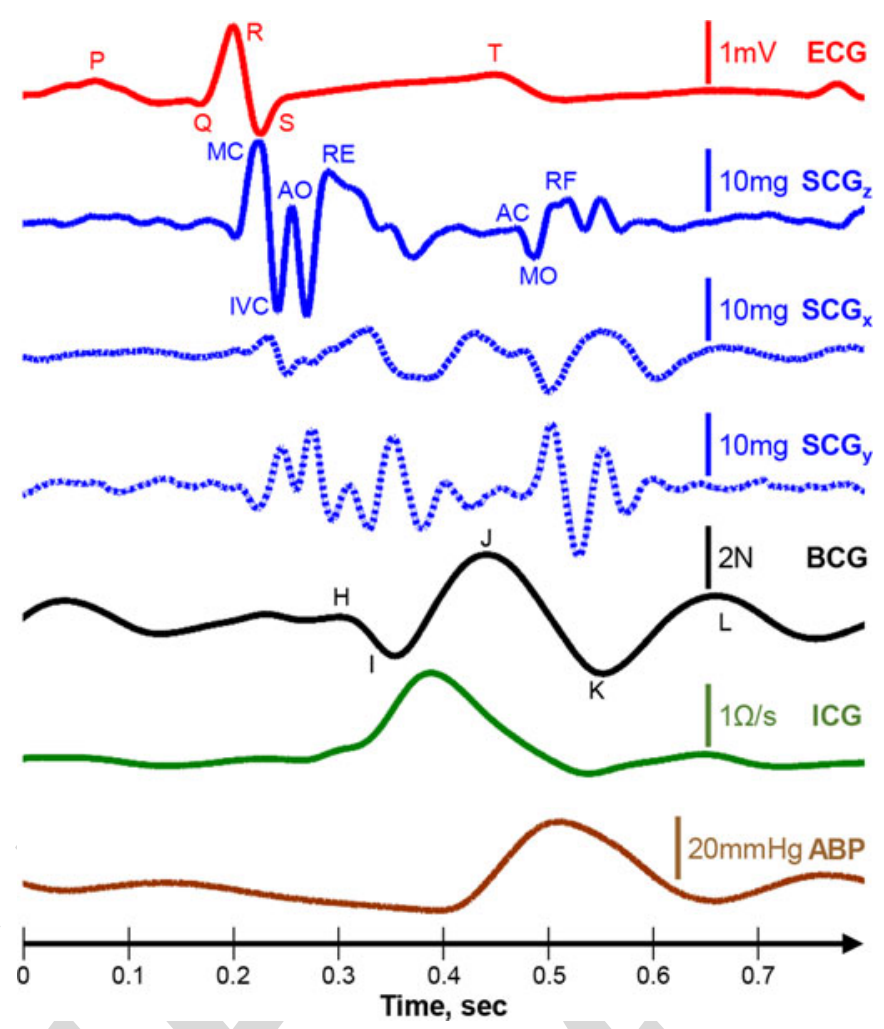

Fig. 1. Simultaneously acquired Lead II electrocardiogram (ECG); three-axis seismocardiogram (SCG) with $z$ indicating the dorso-ventral axis, $x$ indicating the right-to-left lateral axis, and $y$ indicating the head-to-foot axis; ballistocardiogram (BCG); impedance cardiogram (ICG); and arterial blood pressure $(\mathrm{ABP})$ measured at the finger, signals from one subject, illustrating the relative timing and amplitude features of the signals.

leys reflecting specific events of the beating heart. Fig. 1 shows a typical ECG, head-to-foot BCG, tri-axial SCG, impedance cardiogram (ICG), and arterial blood pressure (ABP) measurement from a healthy subject (data were collected with approval from the Institutional Review Board, IRB, at the Georgia Institute of Technology, and with written informed consent obtained). A high-resolution, miniature accelerometer was used for the SCG data collection (356A32, PCB Piezotronics, Depew, NY, USA), and a modified weighing scale was used for the BCG recording as described previously in [15]. The ECG and ICG waveforms were measured using the BN-RSPEC and BN-NICO wireless units (BIOPAC Systems, Inc., Goleta, CA, USA) interfaced to the MP150WSW data acquisition hardware (BIOPAC Systems, Inc., Goleta, CA, USA). The ABP was measured from the finger using the A2SYS Nexfin Monitor (Edwards Lifesciences, Irvine, CA, USA). For this measurement, $z$ corresponded to the dorso-ventral, $y$ to the head-to-foot, and $x$ to the right-to-left lateral components of the SCG. The labels of the peaks and valleys of the dorso-ventral components shown in this figure are according to [16], [17]; for the BCG, the labels are according to [1]. For the SCG, the labels correspond to the physiological event they are believed to represent: MC, mitral valve closure; IVC, isovolumetric contraction; AO, aortic valve opening; RE, rapid ejection; $\mathrm{AC}$, aortic valve closure; $\mathrm{MO}$, mitral valve opening; and RF, rapid filling. For the BCG, the labels of the waves are not associated directly with underlying events, but rather the current understanding is that the waveform represents the 

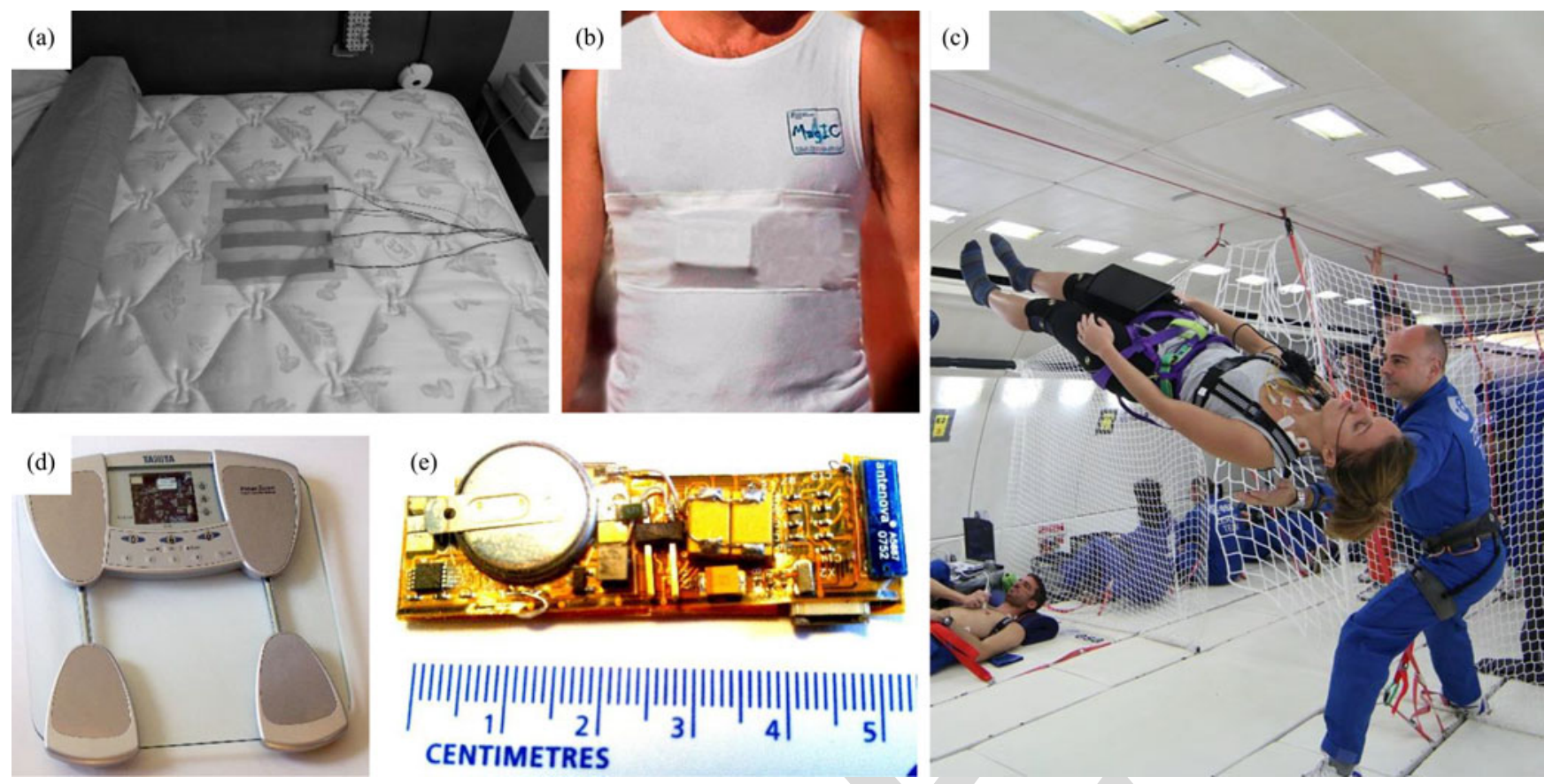

Fig. 2. Compilation of modern BCG and SCG acquisition hardware. (a) PVDF sensor installed into the bed for BCG measurements during sleep. (b) Tri-axial SCG measurement system built into the MagIC-SCG vest for continuous recordings during normal activities of daily living. Modified from [14] with permission. (c) Wearable 3-D BCG measurement hardware (Pneumocard) being used on board a parabolic flight for microgravity BCG measurements; Photo Credit: ESA. (d) Weighing scale with built in circuitry for BCG measurement from a standing subject. (e) Flexible hardware for chest-mounted tri-axial SCG measurements.

combined mechanical pulse response of the vasculature and body to cardiac ejection of blood [18]. Note that, when the BCG is measured by a scale or force plate, the SCG and BCG units are not the same; the SCG records the accelerations of the chest wall, and is thus presented in units of milligram; the BCG represents the displacements of the center of mass of the subject on the weighing scale, which are then converted to units of force by the spring constant for the scale platform, and thus it is presented in units of Newtons. The mass that is accelerated for the SCG is not the same as the mass accelerated for the BCG; as such, the direct conversion of the BCG to acceleration units or the SCG to force units has not yet been elucidated.

\section{Importance of Sensor Location, Axis Selection and Orientation}

For both BCG and SCG, the measurement location has a significant bearing on the morphology, amplitude, and clinically relevant features of the signal. For the SCG, since it is a measure of local vibrations, the precise location of the sensor on the chest impacts the measured signal [19]-[21]. A widely used placement has been on the sternum [14], [22], [23]. Pandia et al. found that the second heart sound was more pronounced when the SCG was measured on the left side of the chest compared to the sternum [19]. For BCG signals measured using an accelerometer, the same is true; an accelerometer placed on the foot will not measure the same BCG signal as one placed on the head, thus stressing the importance of a clear description of, and thoughtfulness regarding, the sensor location on the body.
An additional crucial issue is the orientation of the acceleration axis. BCG or SCG accelerations in the dorso-ventral direction will not be identical to those in the lateral (right-to-left) or headto-foot direction; consequently, depending on the purpose of the measurement the axis should be chosen accordingly or a three-axis accelerometer should be used.

In spite of the major role played by the selection of the measurement axes, the axes orientation, and the sensor location, from the review of the existing literature it appears that information on these aspects is often missing, making difficult the understanding of the experimental setup and the interpretation of results. Thus, as detailed in Section VI, a standardization on these issues is deemed necessary, and in the meantime, it is advisable that the above pieces of information are clearly stated in any scientific communication dealing with BCG and SCG.

\section{INSTRUMENTATION: ENABLING UBIQUITOUS MONITORING}

Fig. 2 shows a compilation of photos depicting several examples of modern BCG and SCG acquisition hardware, enabling data acquisition in a variety of settings, including in bed, in the home, outdoors, and in microgravity. These systems are discussed below in detail.

\section{A. Wearable BCG or SCG Systems}

The primary advantage of wearable BCG or SCG measurement systems is the possibility of obtaining data continuously throughout normal daily living. Additionally, recordings with wearable systems can potentially be acquired in any 
environment; thus, providing an opportunity to assess a person's cardiovascular performance under various environmental settings or stressors.

The sensor type used most often for wearable BCG or SCG measurements is an accelerometer, typically with three-axis measurement capability, that is mechanically coupled to the body with either adhesives, plastic mounting, or textiles. In 2007, Castiglioni et al. tested the SCG assessment by an external three-axis MEMS accelerometer placed on the left clavicle, connected to a smart garment with textile ECG electrodes, thus obtaining simultaneous tri-axial SCG and single-lead ECG recordings [24]. The concept was subsequently refined, and in 2010, Di Rienzo et al. proposed an integrated vest equipped with sensors, the MagIC-SCG device, in which the accelerometer was inside the system electronics and placed in contact with the subject's sternum [14]. Through this system, SCG was recorded over $24 \mathrm{~h}$ in ambulant subjects, while performing a variety of activities of normal daily living and beat-to-beat estimates of cardiac time intervals (CTIs) could be estimated [21]. Chuo et al. developed miniaturized hardware $(55 \times 15 \times 3 \mathrm{~mm})$ on a flexible substrate with adhesive backing for wireless tri-axial SCG recording from the sternum (also with a MEMS accelerometer) together with single-lead ECG and coarse single-point skin temperature via a thermistor [25]. Baevsky et al. developed a portable system, "Pneumocard," for the assessment of the cardiac function of cosmonauts on board the International Space Station [26]. The system comprised a single-axis MEMS accelerometer placed at the apex of the heart for the recording of the SCG signal. Later, a three-axis MEMS accelerometer was added to the system for the recording of the BCG signal. The accelerometer was placed on the back of the subject, either at the center of mass or between the scapulae and its performance during the microgravity phases of parabolic flights was tested by Migeotte et al. [27]-[29].

$\mathrm{He}$ et al. placed a tri-axial MEMS accelerometer for BCG measurement in a plastic mount over the ear, with auxiliary sensors include for ECG and / or photoplethysmogram (PPG) measurement, respectively, [30], [31]. Hyun et al. used an electromagnetic film (EMFi) patch to measure the vibrations of the chest wall in the dorso-ventral direction (transverse); however, it should be noted that the exact position on the chest for the measurement was not provided, and on the basis of morphology, while the signal was called the BCG, it was likely rather an SCG [32]. Another notable approachthat is not exactly a wearable device, but provides some similar advantages-was demonstrated by Balakrishnan et al. with the head-to-foot (longitudinal) direction ballistocardiographic displacements of the head being captured and processed from video recordings [33].

\section{B. Weighing Scale BCG}

The first measurement of BCG on an electronic scale was demonstrated in 1990 by Jim Williams of Linear Technology, as described in his application note AN-43 [34]. Williams built an elegant circuit capable of measuring bodyweight with tremendous accuracy— $4.5 \mathrm{~g}$ resolution up to $136 \mathrm{~kg}$ —and found mo- tion artifacts, and the BCG as the largest sources of noise for 242 his measurements.

The main advantage with weighing-scale-based BCG measurement is that the subject is standing up for the measurementironically, this is also the main disadvantage. While the standing posture of the subject is ideal for ensuring that the measurement is purely longitudinal, it also means the measurements are susceptible to motion artifacts and floor vibrations. This also places a practical limit on the duration of the measurements, as the patient will likely only stand still on the scale for 30-60 s at a time at most. Another key advantage of these systems is that they leverage the tremendous popularity of weighing scales, with more than $80 \%$ of American households owning a scale, and multiple companies developing new and improved "smart" scales with enhanced capabilities. The scale is also used by heart failure patients at home to monitor increasing trends in their bodyweight, which may be related to increased fluid retention [35], [36].

With these potential advantages in mind, researchers have rigorously investigated this mode of BCG measurement. Inan et al. measured the mechanical frequency response of several commercially available scales at various loads to determine if the bandwidth was sufficient for BCG recording over a wide range of bodyweight. For bodyweights up to $160 \mathrm{~kg}$, they found that the mechanical systems of most commercial scales have a bandwidth exceeding $15 \mathrm{~Hz}$, which is sufficient for BCG measurement [15]. Note that for preserving the accuracy of time interval detection from the BCG, such as the R-J interval between the ECG and BCG, analog and digital low-pass filtering operations should not use a cutoff frequency lower than $25 \mathrm{~Hz}$ [37]. BCG measurement on a scale has also been successfully demonstrated by Gonzalez-Landaeta et al. [38] and Shin et al. [39], and in all studies the shape and amplitude of the signal is very similar to the traditional BCG recordings taken by Starr et al. nearly a century earlier [1].

\section{Bed-Based BCG Systems}

BCG can be applied in evaluating the sleep stages and sleep 277 related disorders in more comfortable environment replacing 278 some functions done by polysomnography (PSG). Since BCG- 279 based technology does not require attaching electrodes on pa- 280 tient body surface, it has advantage over ECG of not disturb- 281 ing subject's ordinary sleep behaviors in collecting data. BCG 282 measurement can be integrated with the subject's sleeping en- 283 vironment using several types of sensors, the first of which was 284 a static charge sensitive bed by Alihanka et al. [40], and more 285 recently the following implementations: Pressure sensor in the 286 air mattress [41] or in pad [42], film-type force sensors [43] or 287 load cells in the legs of bed [44], microbend fiber optic BCG 288 sensor [45]-[47], EMFi sensors [48], piezoelectric film sensors 289 [49] or polyvinylidene fluoride (PVDF) sensors [50] in the mat- 290 tress pad, strain gauges [51], pneumatic [52], and hydraulic [53] 291 sensors. Some researchers have also proposed the use of sensor 292 arrays rather than single sensors to improve robustness [54], 293 [55]. As these sensors can usually provide the additional infor- 294 mation on respiration and body movement as well as heart beats, 295 this information can be incorporated with the BCG to generate 296

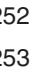
(2) ass

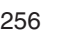
257 258

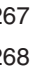
272 273 274

\section{4} .6.

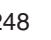

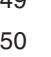

\section{.}

\section{(20)} (t) (a) (a) sis , ex (t) 赵, 党 2t5 
sleep evaluating parameters more accurately, as well as other applications such as early warning in the general ward, or home monitoring, where rhythm and dynamics can be monitored over extended periods of time for predictive analytics.

Sleep stages have mainly been classified into two levels slow wave sleep or non-slow wave sleep (SWS/non-SWS), or three levels (wake/REM/NREM) based on BCG. The earliest implementation of BCG based sleep staging was by Watanabe and Watanabe [56]. Two stage classification between SWS and nonSWS was performed based on BCG with movement measured unobtrusively by a load cell installed bed [44]. Based on calculated heart rate variability (HRV) parameters, they achieved the mean agreement of $92.5 \%$ (kappa index of 0.62). Sleep efficiency was evaluated by detecting nocturnal awakening epochs in BCG measured using PVDF sensors on bed mattress [57], based on the principle that awakening during sleep is related with subtle changes in heart rate; thus, awakening epochs can be detected based on HRV parameters. They achieved the classification accuracy of $97.4 \%$ (kappa index of 0.83 ) and 96.5\% (kappa index of 0.81) and evaluated the sleep efficiency with absolute error of $1.08 \%$ and $1.44 \%$ for normal subjects and obstructive sleep apnea patients, respectively.

Three stage classification (Wake/REM/NREM) of sleep has been derived using the analyses of spectral components of the heartbeats extracted from multichannel BCG based on EMFi sensors [58]. By applying a hidden Markov model only on BCG, they achieved a total accuracy of 79\% (kappa index of 0.43 ) compared to clinical sleep staging from PSG. The performance was enhanced by combining the time variant-autoregressive model (TVAM) and wavelet discrete transform (WDT) with the quadratic (QD) or linear discriminant (LD) analysis [59]. The QD-TVAM algorithm achieved a total accuracy of 76.8\% (kappa index of 0.55), while LD-WDT achieved a total accuracy of 79\% (kappa index of 0.51). Although there was also a study done for sleep stage classification into four levels (wake/REM/deep sleep/light sleep) with ECG [60], four-level sleep stage classification with BCG is not reported yet. With the ECG signal, Tanida et al. classified the sleep stage with HRV analyzed for each 60-s epoch of ECG and calculated at three frequency band powers. Their results for minute-by minute agreement rate ranged from $32 \%$ to $72 \%$ with an average of $56 \%$ for ten healthy women.

Sleep monitoring based on BCG technology has a potential to provide both continuous and longitudinal information on a subjects' sleep quality and may take a role as a predictive screening method prior to the sleep studies based on PSG. It could also fill the gap among PSG of whole night examination and portable ambulatory PSG, which can be applied at home and simplified with, for example, a wrist worn movement sensor.

\section{Chair-Based BCG and SCG systems}

Chair-based systems have mainly used electromechanical film (EMFi) sensors based on piezoelectric transduction. Koivistoinen et al. attached EMFi sensors to a chair to measure BCG signals from two seated subjects, and found the signal shape to be similar to other BCG measurements from the literature
[61]. Walter et al. placed an EMFi mat in the cushion of the driver's seat in a car to measure the BCG for automatically monitoring driver fitness [62]. These systems provide a means for measuring BCG or SCG signals from patients who cannot stand still on their own, minimize motion artifacts, and allow the user to be comfortable during the measurement. The main disadvantages for chair-based BCG recording are the reduction of signal amplitude compared to measurements using table, bed, or weighing scale systems, and the effects of postural changes on signal quality.

\section{Signal PROCESSING AND Modeling}

\section{A. Heartbeat Detection}

Since heart rate is regulated by the autonomic nervous system, the analysis of HRV is currently employed to obtain physiological and clinical information on the level of sympathetic and parasympathetic drive to the heart. Even though ECG is the most widely used biological signal to evaluate heart rate dynamics, BCG may also be used. Due to its easier application for monitoring in contrast to the inconvenience of attaching electrodes to the skin in ECG measurement, BCG may facilitate the assessment of heart rate dynamics in daily life [63].

Heartbeats may be identified by the J-wave peak in the BCG signal, i.e., the point of highest amplitude in the BCG waveform. Heart rate is evaluated by measuring the interval between consecutive J-peaks, the J-J interval. As there are many algorithms to detect the R-peak in ECG, there are also various methods to detect the J-peaks or heart beat from BCG. Since BCG can be measured in different settings with different type of sensors, the peak-detection algorithm should be selected to optimize the performance considering the characteristics of measured BCG. A heartbeat detection algorithm which showed high performance in R-peak detection from ECG can be applied with minor modification for J-peak detection. Generally the peak detection procedure is applied to select the highest value in amplitude as the J-peak within the sliding window after some preprocessing to increase signal-to-noise ratio (SNR) and to reject artifacts due to motion or other interferences.

Choi et al. demonstrated increased detection performance with a dedicated algorithm, which finds local peaks in four divided subintervals within a period and selects the maximum peak as J-peak from these local peaks with some rejection rules [44]. Jansen et al. applied a detection method based on a "template matching" rule by evaluating a correlation function in a local moving window [64], a method which was further refined and developed by Shin et al. [65]. Although this method requires template design in its first stage, Shin et al. successfully applied it to several types of BCG signals acquired from air mattress, load cells, and EMFi sensors. The results showed $95.2 \%$ of sensitivity and $94.8 \%$ of specificity in average for five subjects and three types of BCG signals. Additional methods for heartbeat detection from BCG signals include those which combine different estimators [46], [66], [67], and methods which use wavelets to preprocess the signal prior to peak detection [53], [68].

Heart rate was estimated from the spectral domain specially focusing on third harmonics especially in BCG signals acquired 
with fiber optic sensors [45]. The results showed an error less than 0.34 beat $/ \mathrm{min}$ in $2^{\circ} \mathrm{min}$ averaged heart rate. Heartbeat intervals were calculated with the cepstrum method, by applying FFT for short time windows including pair of consequent heart beats [48]. Relative error of the method was $0.35 \%$ for 15 night recordings with six normal subjects after rejecting movement artifacts. Since the results of heart beat detection are not perfect, generally visual editing is required to correct the errors in peak detection for further application like HRV analysis. Multichannel fusion techniques have also been demonstrated recently for BCG-based heartbeat detection [48], [69].

Recently, Paalasmaa et al. [70] and Brueser et al. [71] both verified heartbeat detection algorithms on large datasets containing hundreds of thousands of heartbeats recorded in uncontrolled environments. Paalasmaa et al. used hierarchical clustering to first infer a heartbeat shape from the recordings, then beat-tobeat intervals were found by determining positions at which this template best fit the signal. The mean beat-to-beat interval error was $13 \mathrm{~ms}$ from 46 subjects in the clinic, home, single bed, double bed, and with two sensor types. Brueser et al. demonstrated robust estimation of heartbeats for 33 subjects of which 25 were insomniacs, with a mean beat-to-beat interval error of $0.78 \%$. Their method used three short-time estimators combined using a Bayesian approach to continuously estimate interbeat intervals. Automatic template learning approaches were also presented by Brueser et al. in 2011 with low error [51].

Performance of HRV analysis using BCG measured on weighing scale-type load cell is evaluated in reference to the ECG during the resting and under each condition of Valsalva and postexercise sessions that induce cardiac autonomic rhythm changes [72]. Time domain, frequency domain, and nonlinear domain HRV parameters were evaluated on 15 healthy subjects to assess the cardiac autonomic modulation under each of these conditions. For all subjects and for all experimental sessions, HRV parameters calculated from BCG peak intervals are statistically not different from those obtained from the reference ECG. The results showed high performance with relative errors of 5.0-6.0\% and strong correlation of 0.97-0.98 in average for these three states compared with the results from ECG peaks. The errors were relatively high in HRV parameters reflecting the high-frequency characteristics of heart rates such as $\mathrm{HF}, \mathrm{LF} / \mathrm{HF}$ in the spectral analysis, pNN50 in time-domain analysis, and SD1 in nonlinear analysis. This is considered to be caused by the inaccuracy in detecting peak from the less sharp J-peak of BCG compared to the R-peak in ECG. HRV estimates with BCG have also been compared to the PPG, and the correlation between the two was found to be high [73]. Preliminary work was recently presented by Brueser et al. for unsupervised HRV estimation from BCG signals [74].

\section{B. Noise and Interference Reduction}

Several sources of noise and interference can potentially corrupt BCG and SCG measurements taken using modern systems. These include sensor and circuit noise [75], motion artifacts [15], [21], [76], [77], and floor vibrations (for standing BCG measurements) [78].
Both BCG and SCG represent low-level signals that contain very low-frequency information - this can lead to problems with flicker (1/f) noise in the sensor interface circuit corrupting the measurements. Furthermore, many diseased subjects, and elderly subjects, have smaller signal amplitudes compared to the healthy young population [79]. The sensor and circuit noise were characterized and reduced for weighing-scale-based BCG systems using an ac-bridge amplifier approach [75]. This approach led to a SNR improvement of $6 \mathrm{~dB}$.

For ambulatory and standing subjects, motion artifacts present the greatest potential obstacle to achieving reliable measurements. Unlike bed or chair systems, where the subject stays generally still for the measurement, postural sway, or ambulation can create unwanted peaks or distortion in the measured signals. Motion artifact detection for standing BCG measurements was accomplished using auxiliary sensors as noise references; then, gating the BCG signal based on the detection of excessive noise [76], [80]. In one study, the noise reference was an extra strain gauge added to the scale to detect postural sway [76]. In another study, the rms power of the electromyogram signal from the feet, indicating the presence of increased muscle contractions due to excessive movement, was used as a noise gate for the BCG [80]. Pandia et al. presented preliminary methods for cancelling motion artifacts in SCG signals from walking subjects, improving overall heartbeat detection [77]. Di Rienzo et al. used an automatic selection of movement-free data segments from daily recordings of SCG signals from ambulant subjects, followed by an ECG triggered ensemble averaging to reduce signal noise [21]. This enabled, for the first time, the assessment of systolic time interval profiles during normal daily living.

BCG measurements taken in a direction orthogonal to the plane of the floor can potentially be corrupted by floor vibrations - this can particularly pose a challenge for measurements taken on a vehicle [62] or plane [81]. Walter et al. instrumented the seat of a car with an EMFi mat to measure the BCG, aiming to use the information to monitor driver fitness [62]. However, with the engine turned on, the BCG was corrupted by vibration artifacts and rendered unusable. Inan $e t$ al. used an auxiliary sensor for vibration detection and adaptive noise cancellation to cancel floor vibration artifacts in the BCG measurement [78]. In this study, high-quality BCG measurements were successfully demonstrated from a subject standing on a bus with the engine turned on and idling. Additionally, it was observed that low-noise SCG waveforms could be obtained in a subject sitting in the metro, while a train was going by, with the above mentioned ensemble averaging approach [21].

\section{Signal Modeling}

Modeling of SCG and BCG provides a tool to better un- 509 derstand the genesis of waves in these signals and to simulate 510 their morphological changes with different myocardial abnor- 511 malities. Modeling of BCG goes back to the early years of 512 ballistocardiographic research [79].

In most BCG recording systems, the recording device is quite 514 small compared to the human body and the platform on which 515 it rests. It is also far away from the heart in most cases; thus, 516 .

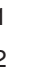
. :5 作 


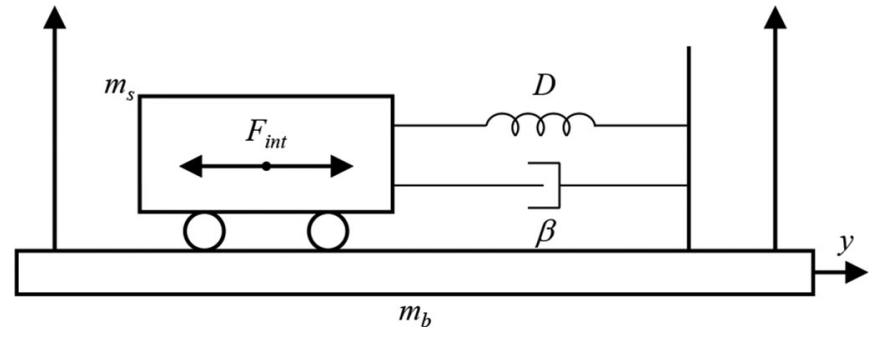

Fig. 3. Schematic showing the subject (with mass, $m_{s}$ ) and the BCG recording system (with mass, $m_{b}$ ) coupled by a spring dashpot system.

TABLE II

DESCRIPTIONS OF VARIABLES FOR SIGNAL MODELING

\begin{tabular}{lc}
\hline \hline Variable & Description \\
\hline$F_{\text {int }}$ & Internal forces \\
$\beta$ & Damping constant \\
$y$ & Displacement or (in subscript) indicating \\
$\dot{y}$ & head-to-foot direction \\
$\ddot{y}$ & Velocity \\
$D$ & Acceleration \\
$m_{s}$ & Spring constant \\
$m_{b}$ & Mass of subject \\
\hline \hline
\end{tabular}

the volume of the heart has been neglected in such models. The heart has been modeled like a point source providing the flow to the circulation system model [82]. Such a model is in accordance with the classical definition of BCG to be resulted through movement of center of gravity of the body and platform. On the contrary, in SCG the recording device (i.e., accelerometer) is near the heart and the volume of the heart cannot be neglected in any model dealing with SCG or any other precordial vibration signal. Thus, except for some preliminary efforts [83] SCG modeling has not been pursued by many researchers, probably because of the complications associated with such a model.

In ballistocardiographic research, one can study the events within human body that cause its movement in space, regardless of the recording device or to study the properties of instruments recording them and how their record relates to the movement originating them. Both of these two approaches are briefly introduced.

1) Modeling the Recording Device: During the early years of ballistocardiographic research, several different instruments were used to measure BCGs, from beds hanging from the ceiling [84] to tables strongly coupled to ground [1]. These instruments were giving different records from the same normal subjects. So, efforts were made to model the effect of these instruments on BCG morphology. Limiting ourselves to the head-foot direction the equation giving the components along the $y$-axis (Fig. 3, variables defined in Table II) reads:

$$
\left(F_{\text {int }}\right)_{y}-\beta \dot{y}-D y=\left(m_{s}+m_{b}\right) \ddot{y} .
$$

After sorting and substituting $\left(F_{\text {int }}\right)_{y}$ into $m_{s} \ddot{y}_{c}$ (where $\ddot{y}_{c}$ is the acceleration of center of mass of body):

$$
\left(m_{s}+m_{b}\right) \ddot{y}+\beta \dot{y}+D y=m_{s} \ddot{y}_{c} .
$$

From the above equation, three different classic types of 545 BCGs can be conceived based on the fact that which terms on 546 the left side of the above equation can be neglected. The first is

$$
\left(m_{s}+m_{b}\right) \ddot{y}=m_{s} \ddot{y}_{c}
$$

which means that the movement of bed and body is proportional 548 to the movement of the center of gravity. A good approximation 549 of this special case is when the ballistocardiograph is weakly 550 coupled to the environment such as ultralow frequency BCG (ULF-BCG) systems.

The second type is when:

$$
\dot{y}=\frac{m_{s}}{\beta} \ddot{y}_{c}
$$

which represents Nickersons's low-frequency (LF) BCG and 554 the third type is when:

$$
y=\frac{m_{s}+m_{b}}{\beta} \ddot{y}_{c}
$$

which refers to the situation when BCG is strongly coupled to its environment, which were categorized under high-frequency BCG (HF-BCG). In other words, when the resonance frequency of the BCG platform is much higher than heart frequency, then its displacement is proportional to the internal acceleration of body's center of gravity.

From this theoretical evaluation, it is clear that very different results will be obtained when one records any one aspect of motion such as displacement or acceleration from each of the three ideal types of ballistocardiographs [82]. However, there is a fourth category of classical BCGs, which are the direct body recordings based on AHA consensus paper on BCG terminology [85]. Direct body BCGs were always criticized for their inconsistencies [82].

2) Modeling the Internal Forces: Starr started on BCG modeling, where arteries were segmented into $3-\mathrm{cm}$ long pieces and mass of blood in the aortic segment closest to the aortic valve was multiplied by acceleration, derived from cardiac ejection curve, to calculate force. This was repeated when the blood volume shifted to the next segment [82].

A more comprehensive model of human systemic arterial tree with distributed properties was constructed in early 1960s by Starr and Noordergraaf [82] and was improved later on by Westerhof et al. [86]. This model was based on the fact that, when using ULF systems, in which the body was free to move in space in the head-foot axis, it was observed that the body moved first footward and then headward during the cardiac cycle. This was explained as a movement to counteract the displacement of the blood mass, that, shortly after the onset of systole, is first driven headward out of the heart to distend the great vessels, and later footward, as the pulse wave spreads peripherally and blood accumulates at a great distance from the heart in the more peripheral vessels.

The model divided the arterial tree in 115 segments and calculated the position of the body's center of gravity in the longitudinal direction $y_{c}(t)$, as a function of time, by numerical integration of the products of the excess masses of each segment during the interval $t$, and the distance $y_{i}$ between the centre of 
each segment and the reference plane. Noordergraaf's model was successful in quantitatively predicting the amplitudes of ULF BCG waves and in giving an explanation for the origin of the main peaks. The model was verified on the data acquired from an astronaut in MIR station [87], where by using the longitudinal BCG recorded in space the model could be used to derive the aortic flow.

\section{Human SubJects Studies With Modern Systems}

\section{A. Correlation Studies With Healthy Subjects}

Originally, BCG and SCG were proposed as diagnostic tools for the clinic-for example, a patient would lie on a Starr BCG table, the recording would be printed on a strip chart, and the physician would read the recording to make a diagnosis regarding the patient's cardiovascular health [1], [5]. However, the large intersubject variability in the signals hampered this approach, particularly given the limited tools available at that time for signal analysis. On the contrary, studies have shown that the intrasubject variability in the signals over serial measurements is actually low [15] — except in the presence of changing cardiovascular health. For this reason, in the past decade the BCG and SCG have been proposed as tools for monitoring changes in the same patient's health overtime. Then, the subject is his/her own control, and intersubject variability is no longer an obstacle.

To uncover the clinical relevance of BCG and SCG signal features, and to pave the way for future studies with clinical populations, several researchers conducted human subjects studies with a healthy population using modern instrumentation and analysis tools. These studies were mainly designed with a noninvasive protocol for altering the hemodynamics and timing intervals of the heart—such as exercise, Valsalva maneuver, whole-body tilt testing, or lower body negative pressure (LBNP) - then, comparing the changes in the BCG or SCG waveform to changes in a reference standard measurement, such as impedance cardiography (ICG) or Doppler ultrasound.

For both BCG and SCG signals the amplitude (or rms power) components have been shown to modulate with changes in left ventricular function - in particular, changes in stroke volume (SV) or cardiac output (CO). Castiglioni et al. measured clavicular SCG signals before and immediately after exercise and compared the percent changes in the peak-to-peak amplitude of the SCG to changes in $\mathrm{CO}$ as measured by the finometer model flow method, finding a strong correlation for four data points taken from four subjects [24]. Inan et al. further demonstrated that the changes in rms power resulting from exercise, measured during $10 \mathrm{~min}$ of recovery time, were strongly correlated to changes in CO measured by Doppler ultrasound for 275 data points taken from nine subjects [88]. Tavakolian etal. trained a neural network to estimate SV from SCG parameters and tested this classifier on a separate testing dataset, finding an average correlation coefficient of 0.61 , and Bland-Altman agreement limits $(95 \%$ confidence) of $+7.4 \mathrm{~mL},-7.6 \mathrm{~mL}$ for 4900 heartbeats analyzed from eight participants [16]. It is important to note that these error bands are larger than what would be needed for absolute volume estimation using the SCG; however, this may be of interest for future research.
Many researchers have also examined the time intervals both within the signals themselves, and between BCG / SCG signal features and other physiological measurements (e.g., ECG or PPG), to form a relationship between these timing intervals to more well-known parameters [e.g., preejection period (PEP), pulse transit time (PTT), or left ventricular ejection time (LVET)]. The time interval between the ECG R-wave peak and the BCG J-wave peak has been proposed as a surrogate for the PEP - a measure of the IVC period of the heart and an index of cardiac contractility [30], [89]. These authors used the Valsalva maneuver and/or whole body tilt testing to modulate the PEP by changing the autonomic balance between parasympathetic and sympathetic drive, and compared the R-J interval to the PEP measured using ICG. Etemadi et al. demonstrated a strong correlation $\left(R^{2}=0.86\right)$ between the R-J interval and the PEP for 2126 heartbeats across ten subjects performing the Valsalva maneuver [89]. He et al. showed similar results for one example subject with both the Valsalva maneuver and whole-body tilt testing [30]. Tavakolian etal. proposed the interval between the ECG Q-wave and the SCG AO-point as a surrogate for PEP, and found strong correlation between this interval and PEP measurement using ICG and Doppler ultrasound in 25 subjects [16].

Researchers have also attempted to extract data from the BCG relating to blood pressure (BP), leveraging the known relationship between pulse wave velocity estimated using PTT, and Pinheiro et al. suggested the use of BCG and PPG for PTT estimation [90]. Shin et al. compared the R-J interval of the BCG, modulated using the Valsalva maneuver, to beat-by-beat systolic BP (SBP) measurements taken using the Finapres system, finding a strong correlation [39]. Nevertheless, Casanella et al. found that, in case of hemodynamic changes induced by paced respiration, this correlation between R-J interval and SBP was dependent on the subject and was not always observed [91]. Winokur et al. found, for one example subject, that the time interval between the BCG and the PPG signal, both measured at the ear, were correlated to PTT, and could thus be used to estimate BP [31].

Another important interval is the duration of systolic ejection, the LVET, as it provides an indication of what percentage of the cardiac cycle is being devoted to ejection compared to filling. Tavakolian et al. used LBNP to simulate hemorrhage, and found that LVET measurements taken using SCG were significantly different at various stages of LBNP, and correlates with the LBNP levels ( $R=0.90)$ for 32 subjects [92]. Di Rienzo et al. found that with exercise LVET changes measured using wearable SCG are in line with the changes reported in the literature and obtained by traditional laboratory techniques [21], [93].

\section{B. Clinical Findings From Patients With Cardiovascular Disease}

Modern ballistocardiography and seismocardiography sys- 698 tems may be capable of monitoring slow, longitudinal changes 699 in cardiac function associated with a number of cardiovascu- 700 lar diseases. Timely noninvasive detection of subtle changes in 701 cardiac pathophysiology may one day enable daily drug dosage 702 adjustments, thus reducing costly and morbid rehospitalizations 703

\section{9} 650 651 652 653 654 655 656 657 658 659 660 661 662

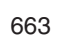

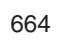
(6) 666 667 668 669 670 671 672 673 674 675 676 677 684 685 to arr

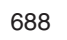

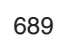
$\infty$

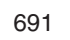
an 693 694 8

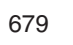
680 681 682

\section{3} 695 
[94]. At this moment, the feasibility of this approach is investigated by the ongoing LAPTOP-HF study which, however, uses an implantable right atrial pressure sensor coupled to a mobile device that allows daily automatic dosage adjustment [95].

Fortunately, the basis for the SCG's clinical utility was begun in 1990 with the initial use of high sensitivity, LF accelerometers to measure precordial vibrations [96]. Significant features of the SCG waveform were identified and associated with key events in the cardiac cycle [17]. This allowed the accurate measurement of these features (e.g., ACs and MOs) using one sensor, greatly simplifying the calculation of CTIs.

A large body of work exists on the utility and efficacy of CTIs [97], [98]. This knowledge combined with the ability to make accurate, repeatable quantitative measurements using the SCG resulted in the ability to conduct clinically relavent crosssectional studies. Subsequently, clinical studies were undertaken to determine if the SCG could be used to identify changes in the SCG waveform resulting from myocardial ischemia [99].

The SCG's clinical utility in enhancing the diagnostic outcome of a graded exercise stress test was first shown in [100]. A large multicenter study demonstrated that when the combined results of the ECG and SCG were used, the predictive accuracy of detecting physiologically significant coronary artery disease was increased significantly over the results of the ECG alone [7].

The introduction in the early 1990s of lightweight $(<25 \mathrm{~g})$ accelerometers, whose working range extended below $1 \mathrm{~Hz}$, made possible other clinical settings for the SCG. The SCG as a magnetic-field-compatible alternative to the electrocardiogram for cardiac stress monitoring [101] was made possible using a newly introduced light weight piezoelectric accelerometer (336C, PCB Piezotronics, Depew, NY, USA).

The SCG was used to measure CTI's during atrial, ventricular, and biventricular pacing, as compared to normals [102]. One of the studies objectives was to determine the utility of the SCG in cardiac resynchronization therapy (CRT). This study was the first to use 3 SCG traces for analysis, i.e., one accelerometer was placed on the xyphoid process, a second over the apex at the fourth intercostal, and a third on the right carotid pulse.

In 1994, the SCG was used to make accurate longitudinal measurements in a study of the effects of elgodiphine on cardiac hemodynamics [103]. In a sports medicine application, exercise capacity was evaluated using the SCG [104]. A more extensive review of the SCG is available in [105].

As a note of interest, the combined patient population of the myocardial ischemia studies [7], [100] is close to 2000 and consists of both healthy and disease subjects. All the raw data were recorded with the same instrumentation (SCG 2000, SeisMed Instruments, Minneapolis, MN, USA) associated with these datasets are complete patient demographics. A project is underway to make the raw data available on the PhysioNet website for study by interested researchers [105].

More recent findings with BCG and SCG further support that the signals have great potential in allowing proactive cardiac disease management without a costly implantable device. However, despite stated clinical and/or physiologic motivations, the overwhelming majority of modern BCG/SCG findings continue to be from healthy subjects [106]-[108]. Notable exceptions in- clude a bed-mounted BCG system for automated detection of 761 atrial fibrillation [109], the observation of reduced signal ampli- 762 tude in the setting of premature atrial or ventricular contractions 763 [15], and the reduction of signal consistency in heart failure 764 patients concordant with worsening clinical outcome [110]. $\quad 765$

One particular subset of patients is particularly well suited for 766 study using cardiomechanical signals, those undergoing CRT. 767 CRT patients have abnormal cardiac conduction causing in a 768 significant delay between the pumping action of the various 769 chambers of the heart. CRT involves precisely adjusting the 770 timing of a multichamber pacemaker to reduce or remove these 771 delays. Such timing is difficult to ascertain using available tech- 772 nologies, spawning the field of "CRT optimization." Researchers 773 recently demonstrated the benefits of intracardiac acceleration 774 monitoring in performing CRT optimization [111], a finding 775 preliminarily corroborated by BCG findings as well [8].

\section{3-D Ballistocardiography and Microgravity Studies}

As the sections on instrumentation earlier in this review have indicated, measurements of BCG (in particular) are constrained by the coupling of the body to the ground, a direct result of the influence of gravity. As such, full 3-D recordings of the BCG are difficult in the terrestrial environment, and much of the focus has been on accelerations in the coronal plane (the $X Y$ plane as defined in the section on measurement axes).

Given this limitation, it is therefore not surprising that the idea of measuring the BCG in a subject in free-fall (weightlessness, zero-G, microgravity) was an obvious target of investigation. The first such experiment was performed in the 1960s in parabolic flight, with the subject strapped into a "tub," which was itself instrumented to record the BCG [9]. Despite the limited periods of microgravity available (typically $\sim 20 \mathrm{~s}$ ) and the subject restraints, recordings of good quality were obtained.

Spaceflight represents the other obvious environment in which the "true" 3-D BCG can be recorded. The earliest recordings were made by the Soviets on Saluyt-6 [10] and consisted of a series of five recordings were performed in two crew members of a long duration mission on days 46, 71, 98, 133, and 175. A piezoelectric sensor, attached close to the center of mass, recorded ballistic forces in the feet-to-head axis during breath holding experiments. Individual changes were seen during the mission with maximum amplitude of the IJ wave occurring on day 133. Measurements were also made during the Spacelab-1 mission aboard the Space Shuttle in 1983 [112]. These experiments were conducted in two subjects at two occasions during this short duration spaceflight and showed an increase of the overall systolic accelerations along the longitudinal axis in microgravity.

Perhaps the best-analyzed dataset of the BCG in spaceflight came from measurements made during the Spacelab D-2 mission in 1993. During that flight, extra time became available (due to an extension of the overall mission length), and an experiment was hastily conceived, approved, implemented, and performed to measure 3-D BCG in a free-floating subject. Parenthetically, this may be one of the fastest spaceflight experiments ever developed with the time from concept, to collection of the data

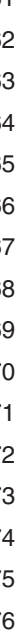




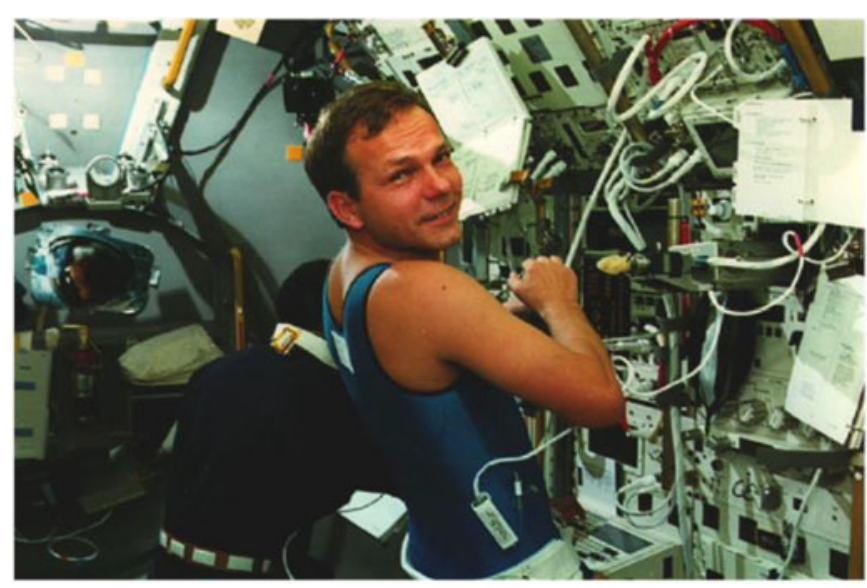

Fig. 4. Subject in D-2 shown wearing the snuggly-fitting suit incorporating a respiratory inductance plethysmograph and ECG. Photo Credit: NASA.

(including approval of an institutional review board) was only 4-5 days, surely some sort of record. The experiment utilized data from a free-floating subject instrumented with an ECG and wearing a snuggly fitting suit that measured respiratory motion using an impedance plethysmograph (see Fig. 4). This instrumentation was a part of the Anthrorack series of human studies managed by the European Space Agency. The second cruicial piece of instrumentation was a set of high-fidelity triaxial accelerometer that were attached to the vehicle and used for measuring the accelerations imparted by crew activity in the Spacelab. The sensor package was detached from the vehicle and taped to the lumbar region of the subject, near to the (presumed) center of mass. Data were then recorded as the subject remained stationary and free floated in the center of the Spacelab, providing a continuous recording, free of interruptions of $146 \mathrm{~s}$. In order to synchronize the two separate data streams, collisions with the Spacelab structure, which disrupted signals in both data streams, were used as posthoc event source [11].

The data from the D-2 study and some subsequent studies provided valuable insight into several aspects of the BCG. In particular there were four major conclusions derived from this dataset.

1) Lung volume greatly influences the accelerations recorded, especially in the longitudinal (head-to-foot) body axis (see Fig. 5), with the implication being that there is better coupling between the heart and the body in the longitudinal axis at higher lung volumes [11]. Interestingly, the actual direction of respiratory motion (mid inspiration versus mid expiration) had only minimal influence of the BCG.

2) Data derived from short periods of microgravity in parabolic flight are largely equivalent to data obtained in sustained microgravity [113].

3) The BCG has a plane of symmetry that is primarily sagittal. This suggests that $2-\mathrm{D}$ recordings performed in a supine subject (i.e., coronal recordings) fail to capture a significant portion of the effect of the blood ejection on the body, complicating their interpretation [113].
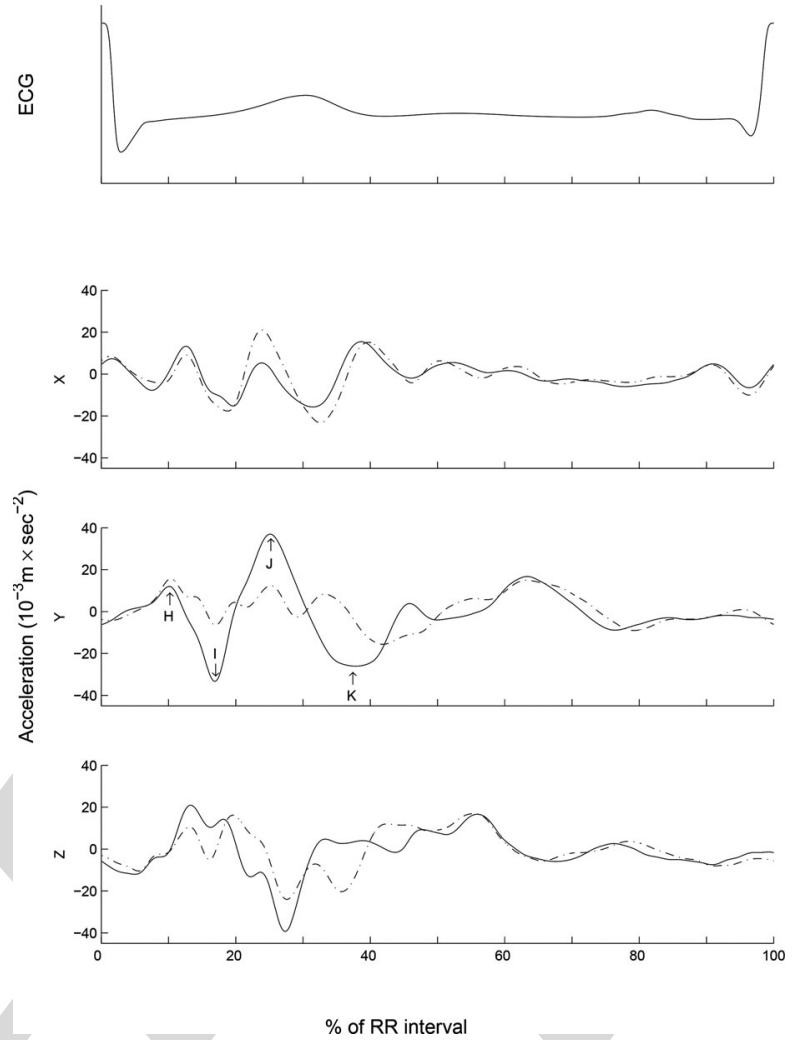

Fig. 5. The 3-D BCG recorded in spaceflight in a free-floating subject, at the end of a normal expiration (dashed lines, functional residual capacity, FRC), and at the end of a normal inspiration (solid lines, FRC + tidal volume). From [11].

4) The accelerations that are recording in a 2-D system are 855 only modestly correlated with the true 3-D accelerations 856 that actually occur, again complicating their interpretation 857 [113].

BCG flight experiments were also an integral part of the 859 Russian cardiovascular research program for the orbital sta- 860 tion MIR. BCG along the head-to-foot direction was measured 861 in three crew members during the second MIR mission in 862 1988 and compared to SCG recordings. Significant changes 863 of the BCG amplitudes (HI, IJ, JK) during the long-term flight 864 were described together with large inter individual differences. 865 The first true 3-D-BCG recordings were made during the sixth 866 MIR mission in 1990 in two crew members on flight days 56867 and 110. Three new piezoelectric sensors were used placed 868 in perpendicular planes in a small cylindrical box with a di- 869 ameter of $40 \mathrm{~mm}$ and a height of $20 \mathrm{~mm}$. The sensitivity of 870 the sensor was $20 \mathrm{mV} / \mathrm{m} / \mathrm{s}^{2}$. The sensor was placed between 871 the scapulae using rubber belts and a metallic plate. The spe- 872 cial amplifier (BCG-3) was connected to the recording unit 873 "Gamma-1," and the data were transmitted telemetrically to 874 the ground station. In summary, no dramatic changes in the vec- 875 tor sum were detected. Maximum forces ranged from 5.85 to 876 10.18 N. However, profound individual changes of the shape, 877 amplitude, and timing of the BCG, especially in the lateral 878 and dorso-ventral plane have been found. Finally, combined 879 BCG and SCG measurements have been made every month 880 in space during the 14 months space flight of Valeri Poljakov, 881 
15th to 17th MIR missions (Russian-Austrian flight experiment "Pulstrans") [114].

\section{STANDARDS AND OPEN ISSUES}

\section{A. Need For a Standardization}

From the analysis of the literature, it appears that important methodological aspects concerning BCG and SCG analysis are still characterized by a certain level of ambiguity. These include

1) Definitions of BCG and SCG Signals: In the literature, the definition of BCG and SCG is not univocal and the "BCG" term is even sometimes used for SCG signals.

2) Nomenclature: Since BCG and SCG waveforms are mostly different (although they might have some common features to be investigated) it is reasonable to use a specific nomenclature for defining peaks and valleys of each signal. The prevalent annotation for BCG was proposed by Starr et al. [1], for SCG by Crow et al. [17]. However, there are some disagreements on these annotations, and in some instances, SCG peaks are termed with the BCG annotation.

3) Indication of Site of Measurement, Characteristics of sensor, Sensor Axis Orientation: These pieces of information are crucial for data comparison and interpretation, but unfortunately are not invariably reported in scientific communications.

A standardization or at least a common position on the above issues would greatly facilitate the understanding and comparison of published results, the exchange of data, and the design of new experimental protocols in this area.

\section{B. Open Issues}

A number of open issues remain to be addressed in this field to improve the understanding and applicability of BCG and SCG signals. Hereafter, we provide just a short list of these issues.

1) The biological meaning of BCG and SCG deflections not yet annotated and their clinical relevance.

2) Possible common features of the BCG and SCG signals.

3) Further parameters derivable from the analysis of the BCG and SCG 3-D vectors.

4) Effects of respiration, posture, right ventricle, and sensor adherence on the signal waveform/quality.

5) How to facilitate the use of these signals in clinical practice?

6) Reference values for healthy and diseased subjects for both types of signals, and for a wide range of body types/sizes, and ages.

\section{CONCLUSION AND AREAS FOR FUTURE INVESTIGATION}

The recent advances in the BCG and SCG field indicate the strong potential of these measurements to address wide variety of clinical needs, in particular monitoring or trending the cardiomechanical health of patients outside of the clinic. Both BCG and SCG measurements can be taken using inexpensive and unobtrusive sensors, making them ideally suited, for example, for home monitoring of chronic diseases. Nevertheless, to maximize our ability to interpret these signals, the physiological origins of both signals must be studied further and elucidated. Furthermore, there is a need to be able to map each measurement modality to another using cardiovascular and mechanical modeling of the body, such that any BCG or SCG waveform amplitude, timing, or morphology measured using one modality can be translated quantitatively to another. For example, if a bed-based recording in the dorso-ventral axis yielded a peak BCG J-wave amplitude of $2 \mathrm{~N}$, system modeling tools are needed to compare this to a corresponding J-wave amplitude measured using a weighing scale. Finally, an extensive, open database of BCG and SCG signals, processing tools, and even microprocessor code needs to be made available to massively expand the capability of researchers around the world to investigate these signals, use them in their own settings, and grow the field from a niche into an established technique, routinely used in clinical practice.

\section{REFERENCES}

[1] I. Starr, A. J. Rawson, H. A. Schroeder et al., "Studies on the estimation of cardiac output in man, and of abnormalities in cardiac function, from the heart's recoil and the blood's impacts; the ballistocardiogram," Amer. J. Physiol., vol. 127, pp. 1-28, 1939.

[2] B. S. Bozhenko, "Seismocardiography — a new method in the study of functional conditions of the heart [Article in Russian]," Ter Arkh, vol. 33, pp. 55-64, 1961 .

[3] J. W. Gordon, "Certain molar movements of the human body produced by the circulation of the blood," J. Anat. Physiol, vol. 11, pp. 533-536, 1877.

[4] D. M. Salerno and J. Zanetti, "Seismocardiography for monitoring changes in left ventricular function during ischemia," Chest J., vol. 100 pp. 991-993, 1991.

[5] I. Starr and F. C. Wood, "Studies with the ballistocardiograph in acute cardiac infarction and chronic angina pectoris," Amer. Heart J., vol. 25, pp. 81-101, 1943.

[6] H. Mandelbaum and R. A. Mandelbaum, "Studies utilizing the portable electromagnetic ballistocardiograph: IV. The clinical significance of serial ballistocardiograms following acute myocardial infarction," Circulation, vol. 7, pp. 910-915, 1953.

[7] R. A. Wilson, V. S. Bamrah, J. J. Lindsay et al., "Diagnostic accuracy of seismocardiography compared with electrocardiography for the anatomic and physiologic diagnosis of coronary artery disease during exercise testing," Amer. J. Cardiol., vol. 71, pp. 536-545, 1993.

[8] L. Giovangrandi, O. T. Inan, R. M. Wiard et al., "Ballistocardiography: A method worth revisiting," in Proc. IEEE Annu. Int. Conf. Eng. Med. Biol. Soc., 2011, pp. 4279-4282.

[9] W. C. Hixson and D. E. Beischer, "Biotelemetry of the triaxial ballistocardiogram and electrocardiogram in a weightless environment," Naval School Aviat. Med., Pensacola, FL, USA, 1964.

[10] R. M. Baevsky and I. I. Funtova, "Ballistocardiographic examinations of the salyut-6 fourth expedition crew members," Komsich Biologiya Aviak Medit, vol. 16, pp. 34-37, 1982.

[11] G. K. Prisk, S. Verhaeghe, D. Padeken et al., "Three-dimensional ballistocardiography and respiratory motion in sustained microgravity," Aviat. Space Environ. Med., vol. 72, pp. 1067-1074, 2001.

[12] P. F. Migeotte, S. De Ridder, J. Tank et al., "Three dimensional ballistoand seismo-cardiography: HIJ wave amplitudes are poorly correlated to maximal systolic force vector," in Proc. IEEE Annu. Int. Conf. Eng. Med. Biol. Soc., 2012, pp. 5046-5049.

[13] R. M. Wiard, H. J. Kim, C. A. Figueroa et al., "Estimation of central aortic forces in the ballistocardiogram under rest and exercise conditions," in Proc. IEEE Annu. Int. Conf. Eng. Med. Biol. Soc., 2009, pp. 2831-2834.

[14] M. Di Rienzo, P. Meriggi, F. Rizzo et al., "A wearable system for the seismocardiogram assessment in daily life conditions," in Proc. IEEE Annu. Int. Conf. Eng. Med, Biol. Soc., 2011, pp. 4263-4266.

[15] O. T. Inan, M. Etemadi, R. M. Wiard et al., "Robust ballistocardiogram acquisition for home monitoring," Physiol. Meas., vol. 30, pp. 169-185, 2009. 
[16] K. Tavakolian, "Characterization and analysis of seismocardiogram for estimation of hemodynamic parameters," Ph.D. dissertation, Dept. Appl. Sci, School Eng. Sci., Simon Fraser Univ., Burnaby, BC, Canada, 2010.

[17] R. S. Crow, P. Hannan, D. Jacobs et al., "Relationship between seismocardiogram and echocardiogram for the events in the cardiac cycle," Amer. J. Noninvasive Cardiol, vol. 8, pp. 39-46, 1994.

[18] O. T. Inan, M. Etemadi, R. M. Wiard et al., "Novel methods for estimating the ballistocardiogram signal using a simultaneously acquired electrocardiogram," in Proc. IEEE Annu. Int. Conf. Eng. Med. Biol. Soc., 2009 , pp. 5334-5347.

[19] K. Pandia, O. T. Inan, G. T. A. Kovacs et al., "Extracting respiratory information from seismocardiogram signals acquired on the chest using a miniature accelerometer," Physiol. Meas, vol. 33, pp. 1643-1660, 2012.

[20] Y. Chuo and B. Kaminska, "Sensor layer of a multiparameter single-point integrated system," IEEE Trans. Biomed. Circuits Syst., vol. 3, no. 4, pp. 229-240, Aug. 2009.

[21] M. D. Rienzo, E. Vaini, P. Castiglioni et al., "Wearable seismocardiography: Towards a beat-by-beat assessment of cardiac mechanics in ambulant subjects," Autonomic Neurosci., vol. 178, pp. 50-59, 2013.

[22] B. Ngai, K. Tavakolian, A. Akhbardeh et al., "Comparative analysis of seismocardiogram waves with the ultra-low frequency ballistocardiogram," in Proc. IEEE Annu. Int. Conf. Eng. Med. Biol. Soc., 2009, pp. 2851-2854.

[23] M. J. T. Paukkunen, M. T. Linnavuo, and R. E. Sepponen, "A portable measurement system for the superior-inferior axis of the seismocardiogram," J. Bioeng. Biomed. Sci., vol. 3, 2013.

[24] P. Castiglioni, A. Faini, G. Parati et al., "Wearable seismocardiography," in Proc. IEEE 29th Annu. Int. Conf. Eng. Med. Biol. Soc., 2007, pp. 3954 3957.

[25] Y. Chuo, M. Marzencki, B. Hung et al., "Mechanically flexible wireless multisensor platform for human physical activity and vitals monitoring," IEEE Trans. Biomed. Circuits Syst., vol. 4, no. 5, pp. 281-294, Oct. 2010

[26] R. M. Baevsky, I. I. Funtova, A. Diedrich et al., "Autonomic function testing aboard the ISS using 'PNEUMOCARD'," Acta Astronautica, vol. 65, pp. 930-932, 2009.

[27] P. F. Migeotte, Q. Deliere, J. Tank et al., "3D-ballistocardiography in microgravity: Comparison with ground based recordings," presented at the IEEE Eng. Med. Biol. Soc., Osaka, Japan, 2013, pp. 7012-7016.

[28] E. Luchitskaya, Q. Deliere, A. Diedrich et al., "Timing and source of the maximum of the transthoracic impedance cardiogram $(\mathrm{dZ} / \mathrm{dt})$ in relation to the H-I-J complex of the longitudinal ballistocardiogram under gravity and microgravity conditions," presented at the IEEE Eng. Med. Biol. Soc., Osaka, Japan, 2013, pp. 7294-7297.

[29] Q. Deliere, P. F. Migeotte, X. Neyt et al., "Cardiovascular changes in parabolic flights assessed by ballistocardiography," presented at the IEEE Eng. Med. Biol. Soc., Osaka, Japan, 2013, pp. 3801-3804.

[30] D. D. He, E. S. Winokur, and C. G. Sodini, "A continuous, wearable, and wireless heart monitor using head ballistocardiogram (BCG) and head electrocardiogram (ECG)," in Proc. IEEE Annu. Int. Conf. Eng. Med. Biol. Soc., 2011, pp. 4729-4732.

[31] E. S. Winokur, D. D. He, and C. G. Sodini, "A wearable vital signs monitor at the ear for continuous heart rate and pulse transit time measurements," in Proc. IEEE Annu. Int. Conf. Eng. Med. Biol. Soc., 2012, pp. 2724-2727.

[32] E. Hyun, S. Noh, C. Yoon et al., "Patch type integrated sensor system for measuring electrical and mechanical cardiac activities," presented at the IEEE Sensors Applications Symposium (SAS), Queenstown, New Zealand, 2014.

[33] G. Balakrishnan, F. Durand, and J. Guttag, "Detecting pulse from head motions in video," in Proc. IEEE Conf. Comput. Vis. Pattern Recog., 2013, pp. 3430-3437.

[34] J. Williams. 1990, Bridge Circuits: Marrying Gain and Balance. Linear Technology Application Note 43

[35] S. I. Chaudhry, J. A. Mattera, J. P. Curtis, et al., "Telemonitoring in patients with heart failure," N. Engl. J. Med., vol. 363, pp. 2301-2309, 2010.

[36] S. I. Chaudhry, Y. Wang, J. Concato et al., "Patterns of weight change preceding hospitalization for heart failure," Circulation, vol. 116, pp. 1549-1554, 2007.

[37] J. Gomez-Clapers, A. Serra-Rocamora, R. Casanella et al., "Uncertainty factors in time-interval measurements in ballistocardiography," in Proc. 19th IMEKO TC-4 Symp. 17th IWADC Workshop Adv. Instrum. Sens. Interoperability, Barcelona, Spain, 2013, pp. 395-399.

[38] R. Gonzalez-Landaeta, O. Casas, and R. Pallas-Areny, "Heart rate detection from an electronic weighing scale," Phys. Meas., vol. 29, pp. 979-988, 2008.
[39] J. H. Shin, K. M. Lee, and K. S. Park, "Non-constrained monitoring 1075 of systolic blood pressure on a weighing scale," Phys. Meas., vol. 30, 1076 pp. 679-693, 2009.

[40] J. Alihanka, K. Vaahtoranta, and I. Saarikivi, "A new method for long- 1078 term monitoring of the ballistocardiogram, heart rate, and respiration," 1079 Amer. J. Physiol., vol. 240, pp. R384-R392, 1981.

1080

[41] Y. Chee, J. Han, and K. S. Park, "Air mattress sensor system with bal- 1081 ancing tube for unconstrained measurement of respiration and heart beat 1082 movements," Phys. Meas., vol. 26, pp. 413-422, 2005.

[42] D. C. Mack, J. T. Patrie, P. M. Suratt et al., "Development and preliminary 1084 validation of heart rate and breathing rate detection using a passive, 1085 ballistocardiography-based sleep monitoring system," IEEE Trans Inf. 1086 Technol. Biomed., vol. 13, no. 1, pp. 111-120, Jan. 2009.

[43] A. Vehkaoja, S. Rajala, P. Kumpulainen et al., "Correlation approach for 1088 the detection of the heartbeat intervals using force sensors placed under 1089 the bed posts," J. Med. Eng. Technol., vol. 37, pp. 327-333, 2013.

[44] B. H. Choi, G. S. Chung, J.-S. Lee et al., "Slow-wave sleep estimation 1091 on a load cell installed bed: A non-constrained method," Phys. Meas., 1092 vol. 30, pp. 1163-1170, 2009.

1093

[45] Y. Zhu, H. Zhang, M. Jayachandran et al., "Ballistocardiography with 1094 fiber optic sensor in headrest position: A feasibility study and a new 1095 processing algorithm," in Proc. 35th IEEE Annu. Int. Conf. Eng. Med. 1096 Biol. Soc., 2013, pp. 5203-5206.

[46] S. Sprager and D. Zazula, "Heartbeat and respiration detection from 1098 optical interferometric signals by using a multimethod approach," IEEE 1099 Trans. Biomed. Eng., vol. 59, no. 10, pp. 2922-9, Oct. 2012.

[47] L. Dziuda and F. Skibniewski, "Monitoring respiration and cardiac activ- 1101 ity using fiber Bragg grating-based sensor," IEEE Trans. Biomed. Eng., 1102 vol. 59, no. 7, pp. 1934-42, Jul. 2012.

[48] J. M. Kortelainen and J. Virkkala, "FFT averaging of multichannel BCG 1104 signals from bed mattress sensor to improve estimation of heart beat 1105 interval," in Proc. IEEE 29th Annu. Int. Conf. Eng. Med. Biol. Soc., 1106 2007, pp. 6685-6688. 1107

[49] J. Paalasmaa, M. Waris, H. Toivonen et al., "Unobtrusive online moni- 1108 toring of sleep at home," in Proc. IEEE Annu. Int. Conf. Eng. Med. Biol. 1109 Soc., 2012, pp. 3784-3788.

[50] F. Wang, M. Tanaka, and S. Chonan, "Development of a PVDF piezopoly- 1111 mer sensor for unconstrained in-sleep cardiorespiratory monitoring," 1112 J. Intell. Mater. Syst. Struct., vol. 14, pp. 185-190, 2003.

[51] C. Bruser, K. Stadlthanner, S. De Waele et al., "Adaptive beat-to-beat 1114 heart rate estimation in ballistocardiograms," IEEE Trans Inf. Technol. 1115 Biomed, vol. 15, no. 5, pp. 778-786, Sep. 2011.

[52] K. Watanabe, T. Watanabe, H. Watanabe et al., "Noninvasive measure- 1117 ment of heartbeat, respiration, snoring and body movements of a subject 1118 in bed via a pneumatic method," IEEE Trans. Biomed. Eng., vol. 52, 1119 no. 12, pp. 2100-07, Dec. 2005.

[53] X. Zhu, W. Chen, T. Nemoto et al., "Real-time monitoring of respiration 1121 rhythm and pulse rate during sleep," IEEE Trans. Biomed. Eng., vol. 53, 1122 no. 12 , pp. $2553-63$, Dec. 2006.

[54] J. Kortelainen, M. V. Gils, and J. Parkka, "Multichannel bed pressure 1124 sensor for sleep monitoring," presented at the Comput. Cardiol. Conf., 1125 Kraków, Poland, 2012.

[55] C. Brueser, A. Kerekes, S. Winter et al., "Multi-channel optical sensor- 1127 array for measuring ballistocardiograms and respiratory activity in bed," 1128 presented at the IEEE 34th Ann. Int. Conf. Eng. Med. Biol. Soc., San 1129 Diego, CA, USA, 2012.

[56] T. Watanabe and K. Watanabe, "Noncontact method for sleep stage esti- 1131 mation," IEEE Trans. Biomed. Eng., vol. 51, no. 10, pp. 1735-1748, Oct. 1132 2004.

[57] D. W. Jung, S. H. Hwang, H. N. Yoon et al., "Nocturnal awakening 1134 and sleep efficiency estimation using unobtrusively measured ballisto- 1135 cardiogram," IEEE Trans. Biomed. Eng., vol. 61, no. 1, pp. 131-138, Jan. 1136 2014.

[58] J. M. Kortelainen, M. O. Mendez, A. M. Bianchi et al., "Sleep staging 1138 based on signals acquired through bed sensor," IEEE Trans Inf. Technol. 1139 Biomed., vol. 14, no. 3, pp. 776-785, May 2010.

[59] M. Migliorini, A. M. Bianchi, Nistico et al., "Automatic sleep staging 1141 based on ballistocardiographic signals recorded through bed sensors," in 1142 Proc. IEEE Annu. Int. Conf. Eng. Med. Biol. Soc., 2010, pp. 3273-3276. 1143

[60] K. Tanida, M. Shibata, and M. M. Heitkemper, "Sleep stage assessment 1144 using power spectral indices of heart rate variability with a simple algo- 1145 rithm: limitations clarified from preliminary study," Biol.Res. Nursing, 1146 vol. 15, pp. 264-272, 2013.

[61] T. Koivistoinen, S. Junnila, A. Varri et al., "A new method for measuring 1148 the ballistocardiogram using EMFi sensors in a normal chair," in Proc. 1149 IEEE 26th Annu. Int. Conf. Eng. Med. Biol. Soc., 2004, pp. 2026-2029. 1150 
[62] M. Walter, B. Eilebrecht, T. Wartzek et al., "The smart car seat: Personalized monitoring of vital signs in automotive applications," Pers. Ubiquitous Comput., vol. 15, pp. 707-715, 2011.

[63] Y. Lim, K. Hong, K. Kim et al., "Monitoring physiological signals using nonintrusive sensors installed in daily life equipment," Biomed. Eng. Lett., vol. 1, pp. 11-20, 2011.

[64] B. H. Jansen, B. H. Larson, and K. Shankar, "Monitoring of the ballistocardiogram with the static charge sensitive bed," IEEE Trans. Biomed. Eng., vol. 38, no. 8, pp. 748-751, Aug. 1991.

[65] J. H. Shin, B. H. Choi, Y. G. Lim et al., "Automatic ballistocardiogram (BCG) beat detection using a template matching approach," in Proc. IEEE 30th Annu. Int. Conf. Eng. Med. Biol. Soc., 2008, pp. 1144-1146.

[66] D. Friedrich, X. L. Aubert, H. Fuhr et al., "Heart rate estimation on a beatto-beat basis via ballistocardiography - a hybrid approach," presented at the IEEE 32nd Annu. Int. Conf. Eng. Med. Biol. Soc., Buenos Aires, Argentina, 2010.

[67] S. Sprager and D. Zazula, "Optimization of heartbeat detection in fiberoptic unobtrusive measurements by using maximum a posteriori probability estimation," IEEE J. Biomed. Health Informat., vol. 18, no. 4, pp. 1161-1168, Jul. 2014.

[68] W. Xu, W. A. Sandham, A. C. Fisher et al., "Detection of the seismocardiogram W complex based on multiscale edges," presented at the 18th Annu. Int. Conf. IEEE EMBS (EMBC), Amsterdam, The Netherlands, 1996.

[69] C. Bruser, J. M. Kortelainen, S. Winter et al., "Improvement of forcesensor-based heart rate estimation using multi-channel data fusion," IEEE J. Biomed. Health Informat., 2014, to be published.

[70] J. Paalasmaa, H. Toivonen, and M. Partinen, "Adaptive heartbeat modelling for beat-to-beat heart rate measurement in ballistocardiograms," IEEE J. Biomed. Health Informat., 2014, to be published.

[71] C. Brueser, S. Winter, and S. Leonhardt, "Robust inter-beat interval estimation in cardiac vibration signals," Phys. Meas., vol. 34, pp. $123-$ 138, 2013.

[72] J. H. Shin, S. H. Hwang, M. H. Chang, et al., "Heart rate variability analysis using a ballistocardiogram during Valsalva manoeuvre and post exercise," Phys. Meas., vol. 32, pp. 1239-1264, 2011.

[73] X.Zhu, W. Chen, K. Kitamura et al., "Comparison of pulse rate variability indices estimated from pressure signal and photoplethysmogram," in Proc. IEEE Int. Conf. Biomed. Health Informat., 2012, pp. 867 -870.

[74] C. Brueser, S. Winter, and S. Leonhardt, "Unsupervised heart rate variability estimation from ballistocardiograms," presented at the 7th Int. Workshop Biosignal Interpretation, Como, Italy, 2012.

[75] O. T. Inan and G. T. A. Kovacs, "A low noise ac-bridge amplifier for ballistocardiogram measurement on an electronic weighing scale," Phys. Meas., vol. 31, pp. N51-N59, 2010.

[76] R. Wiard, O. Inan, B. Argyres et al., "Automatic detection of motion artifacts in the ballistocardiogram measured on a modified bathroom scale," Med. Biol. Eng. Comput., vol. 49, pp. 213-220, 2011.

[77] K. Pandia, S. Ravindran, R. Cole et al., "Motion artifact cancellation to obtain heart sounds from a single chest-worn accelerometer," presented at the IEEE Int. Conf. Acoust., Speech, Signal Process., Dallas, TX, USA, 2010.

[78] O. T. Inan, M. Etemadi, B. Widrow et al., "Adaptive cancellation of floor vibrations in standing ballistocardiogram measurements using a seismic sensor as a noise reference," IEEE Trans. Biomed. Eng., vol. 57, no. 3, pp. 722-727, Mar. 2010.

[79] I. Starr and S. Ogawa, "On the aging of the heart; why is it so much more conspicuous in the ballistocardiogram than in the pulse?" Amer. J. Med. Sci., vol. 242, pp. 399-410, 1961.

[80] O. T. Inan, G. T. A. Kovacs, and L. Giovangrandi, "Evaluating the lowerbody electromyogram signal acquired from the feet as a noise reference for standing ballistocardiogram measurements," IEEE Trans Inf. Technol. Biomed., vol. 14, no. 5, pp. 1188-1196, Sep. 2010.

[81] R. M. Wiard, O. T. Inan, L. Giovangrandi et al., "Preliminary results from standing ballistocardiography measurements in microgravity," in Proc. IEEE 35th Annu. Int. Conf. Eng. Med. Biol. Soc., 2013, pp. 7290-7293.

[82] I. Starr and A. Noordergraaf, Ballistocardiography in Cardiovascular Research: Physical Aspects of the Circulation in Health and Disease. Philadelphia, PA, USA: Lippincott, 1967.

[83] V. Gurev, K. Tavakolian, J. Constantino et al., "Mechanisms underlying isovolumic contraction and ejection peaks in seismocardiogram morphology," J. Med. Biol. Eng., vol. 32, pp. 103-110, 2012.

[84] Y. Henderson, "The mass movements of the circulation as shown by a recoil curve," Amer. J. Phys., vol. 14, pp. 287-298, 1905.
[85] W. R. Scarborough, S. A. Talbot, J. R. Braunstein et al., "Proposals 1226 for ballistocardiographic nomenclature and conventions: revised and ex- 1227 tended: Report of committee on ballistocardiographic terminology," Cir- 1228 culation, vol. 14, pp. 435-450, 1956.

[86] N. Westerhof, F. Bosman, C. J. De Vries et al., "Analog studies of the 1230 human systemic arterial tree," J. Biomech., vol. 2, pp. 121-134, 1969.1231

[87] J. Palladino, J. P. Mulier, F. Wu, et al., "Assessing in the state of the 1232 circulatory system via parameters versus variables," Cardiovasc. Diagn. 1233 Proc.., vol. 13, pp. 131-139, 1996.

[88] O. T. Inan, M. Etemadi, A. Paloma et al., "Non-invasive cardiac output 1235 trending during exercise recovery on a bathroom-scale-based ballistocar- 1236 diograph," Physiol. Meas., vol. 30, pp. 261-274, 2009.

[89] M. Etemadi, O. T. Inan, L. Giovangrandi et al., "Rapid assessment 1238 of cardiac contractility on a home bathroom scale," IEEE Trans. Inf. 1239 Technol. Biomed., vol. 15, no. 6, pp. 864-869, Nov. 2011.

[90] E. Pinheiro, O. Postolache, and P. Girao, "Pulse arrival time and bal- 1241 listocardiogram application to blood pressure variability estimation," in 1242 Proc. IEEE Int. Workshop Med. Meas. Appl., 2009, pp. 132-136. 1243

[91] R. Casanella, J. Gomez-Clapers, and R. Pallas-Areny, "On time interval 1244 measurements using BCG," in Proc. IEEE Annu. Int. Conf. Eng. Med. 1245 Biol. Soc., 2012, pp. 5034-5037.

1246

[92] K. Tavakolian, G. Houlton, G. A. Durmont et al., "Precordial vibra- 1247 tions provide noninvasive detection of early-stage hemorrhage," J. Shock, 1248 vol. 41, pp. 91-96, 2014.

[93] M. Di Rienzo, E. Vaini, P. Castiglioni et al., "Beat-to-beat estimation of 1250 LVET and QS2 indices of cardiac mechanics from wearable seismocar- 1251 diography in ambulant subjects," in Proc. IEEE 35th Annu. Int. Conf. 1252 Eng. Med. Biol. Soc., 2013, pp. 7017-7020.

[94] S. M. Munir, R. C. Boganev, E. Sobash et al., "Devices in heart fail- 1254 ure: Potential methods for device-based monitoring of congestive heart 1255 failure," Texas Heart Inst. J., vol. 35, pp. 166-173, 2008.

[95] ClinicalTrials.gov. [Online]. Available: http://clinicaltrials.gov/show/ 1257 NCT01121107 1258

[96] J. Zanetti and D. Salerno, "Seismocardiography: A new technique for 1259 recording cardiac vibrations. concept, method, and initial observations," 1260 J. Cardiovasc. Technol., vol. 9, pp. 111-120, 1990. 1261

[97] A. M. Weissler, W. S. Harris, and C. D. Schoenfeld, "Systolic time 1262 intervals in heart failure in man," Circulation, vol. 37, pp. 149-159, 1263 1968.

[98] C. L. Garrard, A. M. Weissler, and H. T. Dodge, "The relationship of 1265 alterations in systolic time intervals to ejection fraction in patients with 1266 cardiac disease," Circulation, vol. 42, pp. 455-462, 1970.

[99] D. M. Salerno, J. M. Zanetti, L. A. Green et al., "Seismocardio- 1268 graphic changes associated with obstruction of coronary blood flow 1269 during balloon angioplasty," Amer. J. Cardiol., vol. 68, pp. 201-207, 1270 1991.

[100] D. Salerno, J. M. Zanetti, L. Poliac et al., "Exercise seismocardiography 1272 for detection of coronary artery disease," Amer. J. Noninvasive Cardiol., 1273 vol. 6, pp. 321-330, 1992.

[101] M. Jerosch-Herold, J. Zanetti, H. Merkle et al., "The seismocardiogram 1275 as magnetic-field-compatible alternative to the electrocardiogram for 1276 cardiac stress monitoring," Int. J. Cardiac Imag., vol. 15, pp. 523-531, 1277 1999.

[102] F. I. Marcus, V. Sorrell, J. Zanetti et al., "Accelerometer-derived time 1279 intervals during various pacing nodes in patients with biventricular pace- 1280 makers: Comparison with normals," Pacing Clin. Electrophysiol., vol. 1281 30, pp. 1476-1481, 2007.

[103] B. Silke, J. Spiers, N. Herity et al., "Seismocardiography during phar- 1283 macodynamic intervention in man," Automedia, vol. 16, pp. 35-44, 1284 1994.

[104] J. R. Libonati, J. Ciccolo, and J. Glassberg, "The tei index and exercise 1286 capacity," J. Sports Med., vol. 41, pp. 108-113, 2001.

[105] J. M. Zanetti and K. Tavakolian, "Seismocardiography: Past, present, and 1288 future," presented at the IEEE Eng. Med. Biol. Soc. Conf., Osaka, Japan, 1289 2013.

1290

[106] E. Pinheiro, O. Postolache, and P. Girao, "Theory and developments in 1291 an unobtrusive cardiovascular system representation: Ballistocardiogra- 1292 phy," Open Biomed. Eng. J., vol. 4, pp. 201-216, 2010.

[107] O. Postolache, P. S. Girao, J. Mendes et al., "Unobstrusive heart rate and 1294 respiratory rate monitor embedded on a wheelchair," in Proc. IEEE Int. 1295 Workshop Med. Meas. Appl., 2009, pp. 83-88.

[108] A. Akhbardeh, K. Tavakolian, V. Gurev et al., "Comparative analysis of 1297 three different modalities for characterization of the seismocardiogram," 1298 in Proc. IEEE Annu. Int. Conf. Eng. Med. Biol. Soc., 2009, pp. 2899- 1299 2903. 
1301 [109] C. Bruser, J. Diesel, M. D. H. Zink et al., "Automatic detection of 1302 Informat., vol. 17, pp. 162-171, 2013.

110] L. Giovangrandi, O. T. Inan, D. Banerjee et al., "Preliminary results from BCG and ECG measurements in the heart failure clinic," in Proc. IEEE Eng. Med. Biol. Soc. Annu. Int. Conf., 2012, pp. 3780-3783.

111] A. I. Hernandez, F. Ziglio, A. Amblard et al., "Analysis of endocardial acceleration during intraoperative optimization of cardiac resynchronization therapy," in Proc. IEEE 35th Annu. Int. Conf. Eng. Med. Biol. Soc., 2013, pp. 7000-7003.

112] A. Scano and M. Strollo, "Ballistocardiographic research in weightlessness," Earth-Orient Appl. Space Technol., vol. 15, pp. 101-104, 1985.
[113] G. K. Prisk and P. F. Migeotte, "Physiological insights from gravity-free 1314 ballistocardiography," in Proc. IEEE 35th Annu. Int. Conf. Eng. Med. 1315 Biol. Soc., 2013, pp. 7282-7285.
[114] R. M. Baevsky, I. I. Funtova, E. S. Luchitskaya et al., "Microgravity: An 1317 ideal environment for cardiac force measurements," Cardiometry Open 1318 Access e-J., vol. 3, pp. 100-117, 2013. 
Q1. Author the abbreviation "EMFi" has been used for two terms, i.e., electromagnetic film and electromechanical film in the 1323 text. Please check.

Q2. Author: Please provide names of all authors in place of et al. in Refs. [1], [7], [8], [11]-[15], [17]-[19], [21], [22], [24]-[29], 1325 [32], [35]-[37], [42]-[45], [49], [51]-[53], [55], [57]-[59], [61]-[63], [65], [66], [68], [69], [72], [73], [76]-[78], [81], [83], 1326 [85]-[89], [92]-[94], [99]-[103], [107]-[111], and [114].

Q3. Author: Please provide the technical report number in Ref. [9].

Q4. Author: Please provide the page range in Ref. [23].

Q5. Author: Please update Refs. [69] and [70], if possible.

Q6. Author: Please provide the year in Ref. [95]. 


\title{
Ballistocardiography and Seismocardiography: A Review of Recent Advances
}

\author{
Omer T. Inan, Member, IEEE, Pierre-Francois Migeotte, Member, IEEE, Kwang-Suk Park, Senior Member, IEEE, \\ Mozziyar Etemadi, Student Member, IEEE, Kouhyar Tavakolian, Member, IEEE, Ramon Casanella, Member, IEEE, \\ John Zanetti, Jens Tank, Irina Funtova, G. Kim Prisk, Senior Member, IEEE, and Marco Di Rienzo, Member, IEEE
}

\begin{abstract}
In the past decade, there has been a resurgence in the field of unobtrusive cardiomechanical assessment, through advancing methods for measuring and interpreting ballistocardiogram (BCG) and seismocardiogram (SCG) signals. Novel instrumentation solutions have enabled BCG and SCG measurement outside of clinical settings, in the home, in the field, and even in microgravity. Customized signal processing algorithms have led to reduced measurement noise, clinically relevant feature extraction, and signal modeling. Finally, human subjects physiology studies have been conducted using these novel instruments and signal processing tools with promising clinically relevant results. This paper reviews the recent advances in these areas of modern BCG and SCG research.
\end{abstract}

Index Terms-Ballistocardiogram (BCG), cardiomechanical signals, noninvasive physiologic monitoring, seismocardiogram (SCG), ubiquitous health.

Manuscript received May 14, 2014; revised August 20, 2014; accepted September 29, 2014. Date of publication; date of current version. The work of P. F. Migeotte was supported by the Belgian Federal Science Policy Office via the European Space Agency PRODEX program (ILSRA-2009-0729). The work of G. K. Prisk was supported by the National Space Biomedical Research Institute through NASA NCC9-58. The work of J. Tank and I. I. Funtova were supported by the German Space Agency (DLR) under Grant 50WB1117. The work of M. Di Rienzo was supported in part by the Italian Space Agency through ASI 2013-061-I.0 and ASI 2013-079-R.0.

O. T. Inan is with the School of Electrical and Computer Engineering, Georgia Institute of Technology, Atlanta, GA 30308 USA (e-mail: oeinan @ gmail.com).

P.-F. Migeotte is with the Department of Cardiology, Universite Libre de Bruxelles 1050, Brussels, Belgium (e-mail: Pierre-Francois.Migeotte@ulb.ac.be).

K.-S. Park is with the Department of Biomedical Engineering, Seoul National University, Seoul 110-799, Korea (e-mail: kspark@ bmsil.snu.ac.kr).

M. Etemadi is with the Department of Bioengineering and Therapeutic Sciences, University of California at San Francisco, San Francisco, CA 94143 USA (e-mail: mozziyar.etemadi@ucsf.edu).

$\mathrm{K}$. Tavakolian is with the Department of Electrical Engineering, University of North Dakota, Grand Forks, ND 58202 USA (e-mail: kouhyart@ gmail.com).

R. Casanella is with the Instrumentation, Sensors, and Interfaces Group, Universitat Politecnica de Catalunya, 08034 Barcelona, Spain (e-mail: ramon. casanella@upc.edu).

J. Zanetti is with Acceleron Medical Systems, Arkansaw, WI 54721 USA (e-mail: jmzsenior@gmail.com).

J. Tank is with the Klinsche Pharmakologie, Medizinische Hochschule Hannover, 30625 Hannover, Germany (e-mail: Tank.Jens@mh-hannover.de).

I. Funtova is with the Laboratory for Autonomic Regulation of Cardiorespiratory System, Institute of Biomedical Problems, Russian Academy of Sciences, 123007 Moscow, Russian (e-mail: funtova.imbp@mail.ru).

G. K. Prisk is with the Department of Medicine and Radiology, University of California at San Diego, La Jolla, CA, 92093 USA (e-mail: kprisk@ucsd.edu).

M. Di Rienzo is with the Department of Biomedical Technology, Fondazione Don Carlo Gnocchi, ONLUS, 20133 Milano, Italy (e-mail: mdirienzo@ dongnocchi.it).

Color versions of one or more of the figures in this paper are available online at http://ieeexplore.ieee.org.

Digital Object Identifier 10.1109/JBHI.2014.2361732

\section{INTRODUCTION}

A $\mathrm{S}$ detailed in the following sections, the ballistocardiogram $(\mathrm{BCG})$ is a measurement of the recoil forces of the body in reaction to cardiac ejection of blood into the vasculature [1], while the seismocardiogram (SCG) represents the local vibrations of the chest wall in response to the heartbeat [2]. The BCG phenomenon was first observed in 1877 by Gordon, with the finding that, as a subject would stand on a weighing scale, the needle would vibrate synchronously to the subject's heartbeat [3]. Nearly 60 years later, Starr and colleagues created an instrument in the form of a table with a mobile top surface to measure the BCG in a repeatable scientific manner [1]. The SCG was first observed by Bozhenko in 1961, and was first applied in clinical studies 30 years later in 1991 by Salerno and Zanetti [4]. Throughout the 1900s, both BCG and SCG signals were heavily investigated and several publications appeared in major scientific and clinical journals (e.g., [4]-[7]). However, because of the advent of echocardiography and magnetic resonance imaging, and overly-cumbersome hardware, BCG and SCG were largely abandoned by the medical community [8].

Today, technological advancements largely simplify the measurement and assessment of these signals and open new perspectives in their clinical use. This paper reviews the instrumentation and signal processing advances which have helped to propel BCG and SCG into this revival. It also summarizes some of the key human subjects studies performed recently that support the use of BCG and SCG in extra-clinical applications.

\section{DESCRIPTION OF BCG AND SCG SIGNALS}

\section{A. BCG Signal Description}

At every heartbeat, the blood travelling along the vascular tree produces changes in the body center of mass. Body micromovements are then produced by the recoil forces to maintain the overall momentum. The BCG is the recording of these movements, can be measured as a displacement, velocity, or acceleration signal, and is known to include movements in all three axes. The longitudinal BCG is a measure of the head-to-foot deflections of the body, while the transverse BCG represents anteroposterior (or dorso-ventral) vibrations. The original bed- and table-based BCG systems focused on longitudinal BCG measurements, representing what was supposed to be the largest projection of the 3-D forces resulting from cardiac ejection [1]. Table I summarizes modern BCG measurement systems and their axes of measurement. Note that for some systems, head-to-foot and dorso-ventral forces are unavoidably, mixed 
TABLE I

MOdERn BCG Systems AND THEIR CORRESPONDING MEASUREMENT AXES

\begin{tabular}{|c|c|c|}
\hline Modern BCG System & Axis & Comments / Challenges \\
\hline Accel. $(0 \mathrm{~g})$ & All (3-D) & - Needs reduced gravity \\
\hline Accel. (1g) & Head-to-foot & $\begin{array}{l}\text { - Placement affects signal shape } \\
\text { and amplitude } \\
\text { - Motion artifacts must be } \\
\text { detected and mitigated }\end{array}$ \\
\hline Bed & $\begin{array}{l}\text { Head-to-foot or } \\
\text { Dorso-ventral }\end{array}$ & $\begin{array}{l}\text { - Cross-axis coupling } \\
\text { - Changes in sleep position affect } \\
\text { signal quality / shape }\end{array}$ \\
\hline Chair & $\begin{array}{l}\text { Head-to-foot or } \\
\text { Dorso-ventral }\end{array}$ & $\begin{array}{l}\text { - Posture affects signal quality } \\
\text { and repeatability }\end{array}$ \\
\hline Weighing Scale & Head-to-foot & $\begin{array}{l}\text { - Posture affects signal quality } \\
\text { and repeatability } \\
\text { - Motion artifacts must be } \\
\text { detected and mitigated }\end{array}$ \\
\hline
\end{tabular}

together in the measurement, and this should be accounted for when interpreting results. However, in spite of the 3-D nature of the BCG, for a long period of time only the microdisplacements of the body along the longitudinal axis (head-to-foot) were considered. Currently, BCG is mainly measured using a force plate or force sensor placed on a weighing scale or under the seat of a chair, with the subject in a vertical position. Modern approaches to BCG measurement are discussed below in Section III.

It should be considered, however, that the gravity force and any contact of the body with external objects, including the floor and measuring devices, somewhat interferes with, or even impedes, the body displacement induced by the recoil forces. As a result, the BCG measurement on earth is always affected by some distortion. The ideal environment for assessing the BCG would be in microgravity settings, such as during space missions. Such experiments have been performed, and the results described below confirm that in microgravity the whole body recoil forces (BCG) are significant in all three dimensions [9]-[12]. Modeling studies examining the cardiogenic traction forces of the aorta have confirmed this finding as well [13].

\section{B. SCG Signal Description}

SCG is the measure of the thoracic vibrations produced by the heart's contraction and the ejection of blood from the ventricles into the vascular tree. Today, the SCG can readily be detected by placing a low-noise accelerometer on the chest. If a tri-axial accelerometer is used, SCG components are present in all three axes, each displaying a specific pattern [12], [14]. However, in the literature, the majority of studies on SCG only focus on the amplitude of the dorso-ventral component, although it is likely that additional biological information could be derived also from the analysis of the longitudinal and lateral SCG components, and from the analysis of the acceleration vector trajectory during the heart cycle. Unless the contrary is stated to be consistent with the prevalent literature only the dorso-ventral acceleration component of SCG will be considered in the remainder of this paper.

\section{BCG and SCG Waveforms}

For each heart contraction, a BCG and SCG waveform is generated. Each waveform is characterized by several peaks and val-

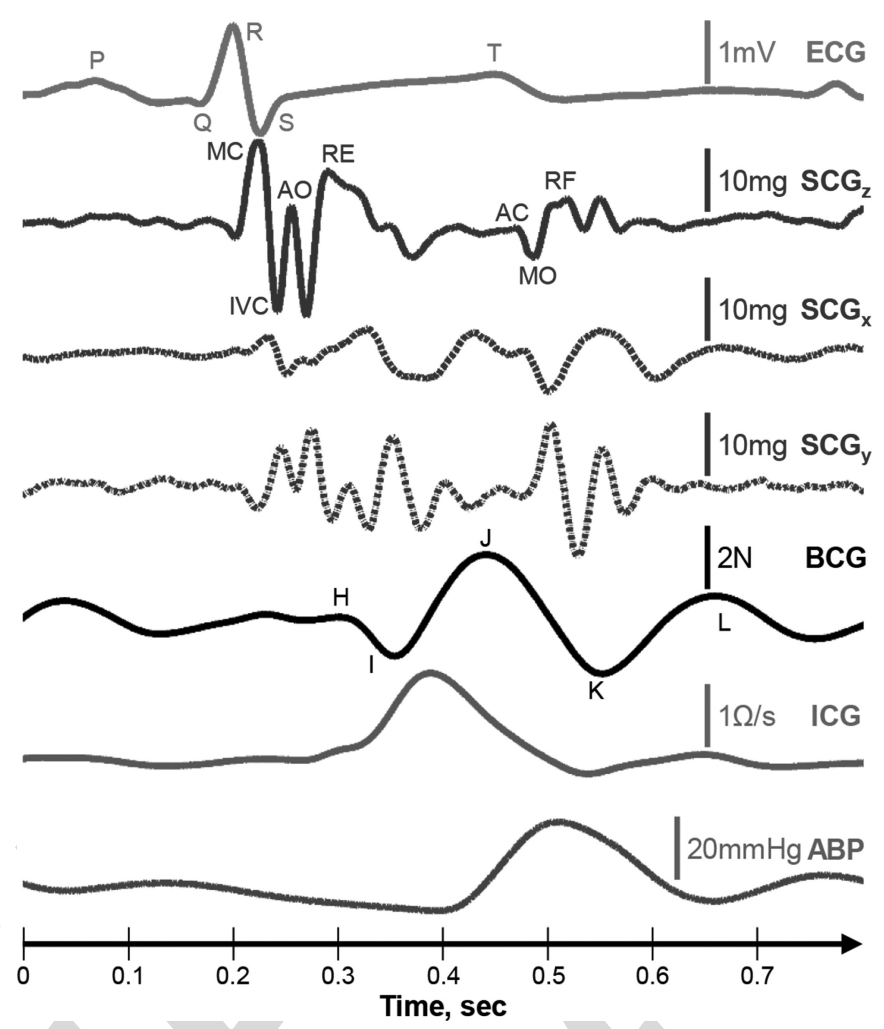

Fig. 1. Simultaneously acquired Lead II electrocardiogram (ECG); three-axis seismocardiogram (SCG) with $z$ indicating the dorso-ventral axis, $x$ indicating the right-to-left lateral axis, and $y$ indicating the head-to-foot axis; ballistocardiogram (BCG); impedance cardiogram (ICG); and arterial blood pressure (ABP) measured at the finger, signals from one subject, illustrating the relative timing and amplitude features of the signals.

leys reflecting specific events of the beating heart. Fig. 1 shows a typical ECG, head-to-foot BCG, tri-axial SCG, impedance cardiogram (ICG), and arterial blood pressure (ABP) measurement from a healthy subject (data were collected with approval from the Institutional Review Board, IRB, at the Georgia Institute of Technology, and with written informed consent obtained). A high-resolution, miniature accelerometer was used for the SCG data collection (356A32, PCB Piezotronics, Depew, NY, USA), and a modified weighing scale was used for the BCG recording as described previously in [15]. The ECG and ICG waveforms were measured using the BN-RSPEC and BN-NICO wireless units (BIOPAC Systems, Inc., Goleta, CA, USA) interfaced to the MP150WSW data acquisition hardware (BIOPAC Systems, Inc., Goleta, CA, USA). The ABP was measured from the finger using the A2SYS Nexfin Monitor (Edwards Lifesciences, Irvine, CA, USA). For this measurement, $z$ corresponded to the dorso-ventral, $y$ to the head-to-foot, and $x$ to the right-to-left lateral components of the SCG. The labels of the peaks and valleys of the dorso-ventral components shown in this figure are according to [16], [17]; for the BCG, the labels are according to [1]. For the SCG, the labels correspond to the physiological event they are believed to represent: MC, mitral valve closure; IVC, isovolumetric contraction; AO, aortic valve opening; RE, rapid ejection; $\mathrm{AC}$, aortic valve closure; $\mathrm{MO}$, mitral valve opening; and RF, rapid filling. For the BCG, the labels of the waves are not associated directly with underlying events, but rather the current understanding is that the waveform represents the 

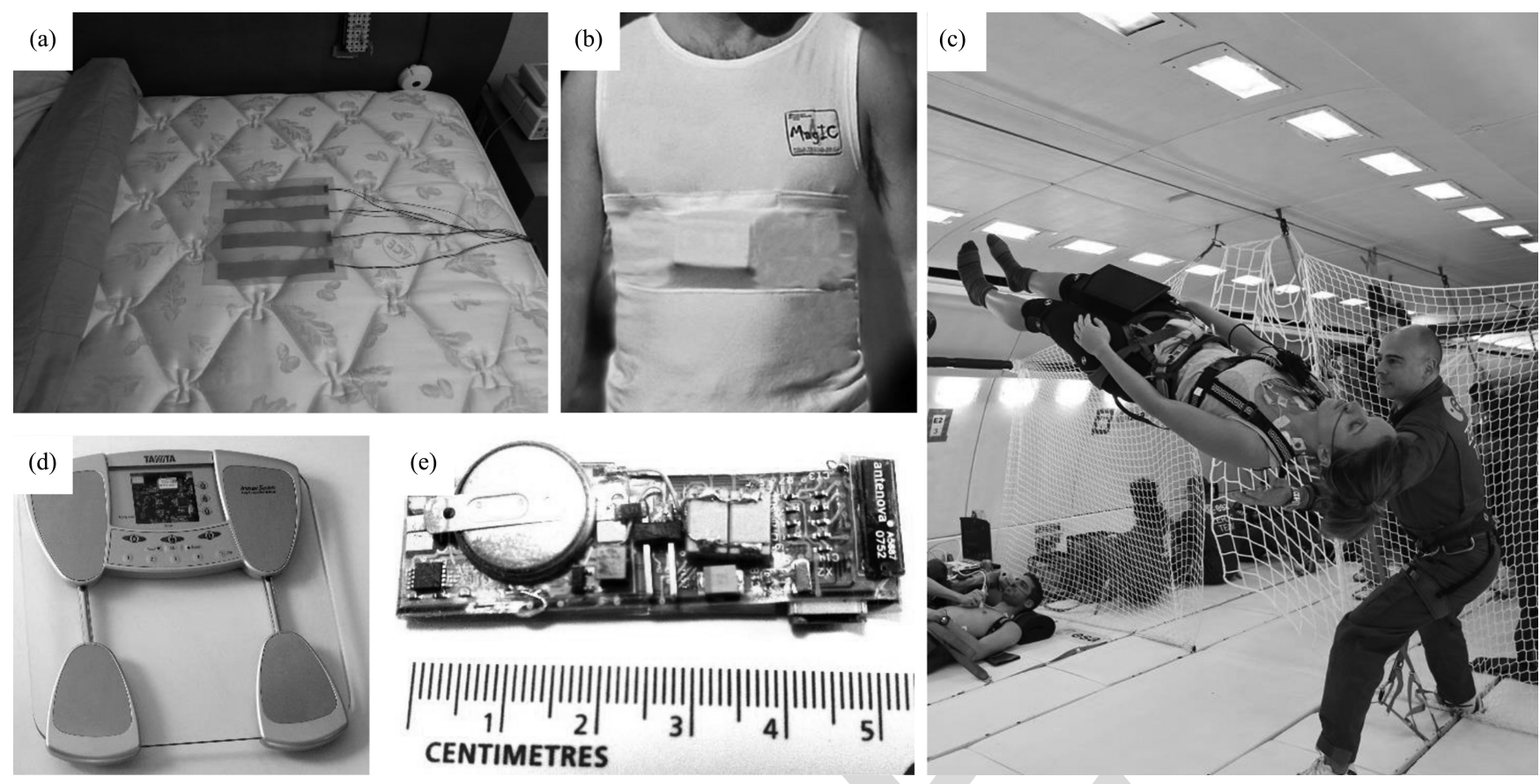

Fig. 2. Compilation of modern BCG and SCG acquisition hardware. (a) PVDF sensor installed into the bed for BCG measurements during sleep. (b) Tri-axial SCG measurement system built into the MagIC-SCG vest for continuous recordings during normal activities of daily living. Modified from [14] with permission. (c) Wearable 3-D BCG measurement hardware (Pneumocard) being used on board a parabolic flight for microgravity BCG measurements; Photo Credit: ESA. (d) Weighing scale with built in circuitry for BCG measurement from a standing subject. (e) Flexible hardware for chest-mounted tri-axial SCG measurements.

combined mechanical pulse response of the vasculature and body to cardiac ejection of blood [18]. Note that, when the BCG is measured by a scale or force plate, the SCG and BCG units are not the same; the SCG records the accelerations of the chest wall, and is thus presented in units of milligram; the BCG represents the displacements of the center of mass of the subject on the weighing scale, which are then converted to units of force by the spring constant for the scale platform, and thus it is presented in units of Newtons. The mass that is accelerated for the SCG is not the same as the mass accelerated for the BCG; as such, the direct conversion of the BCG to acceleration units or the SCG to force units has not yet been elucidated.

\section{Importance of Sensor Location, Axis Selection and Orientation}

For both BCG and SCG, the measurement location has a significant bearing on the morphology, amplitude, and clinically relevant features of the signal. For the SCG, since it is a measure of local vibrations, the precise location of the sensor on the chest impacts the measured signal [19]-[21]. A widely used placement has been on the sternum [14], [22], [23]. Pandia et al. found that the second heart sound was more pronounced when the SCG was measured on the left side of the chest compared to the sternum [19]. For BCG signals measured using an accelerometer, the same is true; an accelerometer placed on the foot will not measure the same BCG signal as one placed on the head, thus stressing the importance of a clear description of, and thoughtfulness regarding, the sensor location on the body.
An additional crucial issue is the orientation of the acceleration axis. BCG or SCG accelerations in the dorso-ventral direction will not be identical to those in the lateral (right-to-left) or headto-foot direction; consequently, depending on the purpose of the measurement the axis should be chosen accordingly or a three-axis accelerometer should be used.

In spite of the major role played by the selection of the measurement axes, the axes orientation, and the sensor location, from the review of the existing literature it appears that information on these aspects is often missing, making difficult the understanding of the experimental setup and the interpretation of results. Thus, as detailed in Section VI, a standardization on these issues is deemed necessary, and in the meantime, it is advisable that the above pieces of information are clearly stated in any scientific communication dealing with BCG and SCG.

\section{INSTRUMENTATION: ENABLING UBIQUITOUS MONITORING}

Fig. 2 shows a compilation of photos depicting several examples of modern BCG and SCG acquisition hardware, enabling data acquisition in a variety of settings, including in bed, in the home, outdoors, and in microgravity. These systems are discussed below in detail.

\section{A. Wearable BCG or SCG Systems}

The primary advantage of wearable BCG or SCG measurement systems is the possibility of obtaining data continuously throughout normal daily living. Additionally, recordings with wearable systems can potentially be acquired in any 
environment; thus, providing an opportunity to assess a person's cardiovascular performance under various environmental settings or stressors.

The sensor type used most often for wearable BCG or SCG measurements is an accelerometer, typically with three-axis measurement capability, that is mechanically coupled to the body with either adhesives, plastic mounting, or textiles. In 2007, Castiglioni et al. tested the SCG assessment by an external three-axis MEMS accelerometer placed on the left clavicle, connected to a smart garment with textile ECG electrodes, thus obtaining simultaneous tri-axial SCG and single-lead ECG recordings [24]. The concept was subsequently refined, and in 2010, Di Rienzo et al. proposed an integrated vest equipped with sensors, the MagIC-SCG device, in which the accelerometer was inside the system electronics and placed in contact with the subject's sternum [14]. Through this system, SCG was recorded over $24 \mathrm{~h}$ in ambulant subjects, while performing a variety of activities of normal daily living and beat-to-beat estimates of cardiac time intervals (CTIs) could be estimated [21]. Chuo et al. developed miniaturized hardware $(55 \times 15 \times 3 \mathrm{~mm})$ on a flexible substrate with adhesive backing for wireless tri-axial SCG recording from the sternum (also with a MEMS accelerometer) together with single-lead ECG and coarse single-point skin temperature via a thermistor [25]. Baevsky et al. developed a portable system, "Pneumocard," for the assessment of the cardiac function of cosmonauts on board the International Space Station [26]. The system comprised a single-axis MEMS accelerometer placed at the apex of the heart for the recording of the SCG signal. Later, a three-axis MEMS accelerometer was added to the system for the recording of the BCG signal. The accelerometer was placed on the back of the subject, either at the center of mass or between the scapulae and its performance during the microgravity phases of parabolic flights was tested by Migeotte et al. [27]-[29].

$\mathrm{He}$ et al. placed a tri-axial MEMS accelerometer for BCG measurement in a plastic mount over the ear, with auxiliary sensors include for ECG and / or photoplethysmogram (PPG) measurement, respectively, [30], [31]. Hyun et al. used an electromagnetic film (EMFi) patch to measure the vibrations of the chest wall in the dorso-ventral direction (transverse); however, it should be noted that the exact position on the chest for the measurement was not provided, and on the basis of morphology, while the signal was called the BCG, it was likely rather an SCG [32]. Another notable approachthat is not exactly a wearable device, but provides some similar advantages-was demonstrated by Balakrishnan et al. with the head-to-foot (longitudinal) direction ballistocardiographic displacements of the head being captured and processed from video recordings [33].

\section{B. Weighing Scale BCG}

The first measurement of BCG on an electronic scale was demonstrated in 1990 by Jim Williams of Linear Technology, as described in his application note AN-43 [34]. Williams built an elegant circuit capable of measuring bodyweight with tremendous accuracy— $4.5 \mathrm{~g}$ resolution up to $136 \mathrm{~kg}$ - and found mo- tion artifacts, and the BCG as the largest sources of noise for his measurements.

The main advantage with weighing-scale-based BCG measurement is that the subject is standing up for the measurementironically, this is also the main disadvantage. While the standing posture of the subject is ideal for ensuring that the measurement is purely longitudinal, it also means the measurements are susceptible to motion artifacts and floor vibrations. This also places a practical limit on the duration of the measurements, as the patient will likely only stand still on the scale for 30-60 s at a time at most. Another key advantage of these systems is that they leverage the tremendous popularity of weighing scales, with more than $80 \%$ of American households owning a scale, and multiple companies developing new and improved "smart" scales with enhanced capabilities. The scale is also used by heart failure patients at home to monitor increasing trends in their bodyweight, which may be related to increased fluid retention [35], [36].

With these potential advantages in mind, researchers have rigorously investigated this mode of BCG measurement. Inan et al. measured the mechanical frequency response of several commercially available scales at various loads to determine if the bandwidth was sufficient for BCG recording over a wide range of bodyweight. For bodyweights up to $160 \mathrm{~kg}$, they found that the mechanical systems of most commercial scales have a bandwidth exceeding $15 \mathrm{~Hz}$, which is sufficient for BCG measurement [15]. Note that for preserving the accuracy of time interval detection from the BCG, such as the R-J interval between the ECG and BCG, analog and digital low-pass filtering operations should not use a cutoff frequency lower than $25 \mathrm{~Hz}$ [37]. BCG measurement on a scale has also been successfully demonstrated by Gonzalez-Landaeta et al. [38] and Shin et al. [39], and in all studies the shape and amplitude of the signal is very similar to the traditional BCG recordings taken by Starr et al. nearly a century earlier [1].

\section{Bed-Based BCG Systems}

BCG can be applied in evaluating the sleep stages and sleep 277 related disorders in more comfortable environment replacing 278 some functions done by polysomnography (PSG). Since BCG- 279 based technology does not require attaching electrodes on pa- 280 tient body surface, it has advantage over ECG of not disturb- 281 ing subject's ordinary sleep behaviors in collecting data. BCG 282 measurement can be integrated with the subject's sleeping en- 283 vironment using several types of sensors, the first of which was 284 a static charge sensitive bed by Alihanka et al. [40], and more 285 recently the following implementations: Pressure sensor in the 286 air mattress [41] or in pad [42], film-type force sensors [43] or 287 load cells in the legs of bed [44], microbend fiber optic BCG 288 sensor [45]-[47], EMFi sensors [48], piezoelectric film sensors 289 [49] or polyvinylidene fluoride (PVDF) sensors [50] in the mat- 290 tress pad, strain gauges [51], pneumatic [52], and hydraulic [53] 291 sensors. Some researchers have also proposed the use of sensor 292 arrays rather than single sensors to improve robustness [54], 293 [55]. As these sensors can usually provide the additional infor- 294 mation on respiration and body movement as well as heart beats, 295 this information can be incorporated with the BCG to generate 296

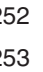
(a) 28 ${ }^{208}$ 257 258

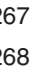
270 271 272 273 273
274 . (2) (1) $\infty$

\section{.}

\section{(as} (t) (a) (a) sis , ex (t) 275 
sleep evaluating parameters more accurately, as well as other applications such as early warning in the general ward, or home monitoring, where rhythm and dynamics can be monitored over extended periods of time for predictive analytics.

Sleep stages have mainly been classified into two levels slow wave sleep or non-slow wave sleep (SWS/non-SWS), or three levels (wake/REM/NREM) based on BCG. The earliest implementation of BCG based sleep staging was by Watanabe and Watanabe [56]. Two stage classification between SWS and nonSWS was performed based on BCG with movement measured unobtrusively by a load cell installed bed [44]. Based on calculated heart rate variability (HRV) parameters, they achieved the mean agreement of $92.5 \%$ (kappa index of 0.62). Sleep efficiency was evaluated by detecting nocturnal awakening epochs in BCG measured using PVDF sensors on bed mattress [57], based on the principle that awakening during sleep is related with subtle changes in heart rate; thus, awakening epochs can be detected based on HRV parameters. They achieved the classification accuracy of $97.4 \%$ (kappa index of 0.83 ) and $96.5 \%$ (kappa index of 0.81) and evaluated the sleep efficiency with absolute error of $1.08 \%$ and $1.44 \%$ for normal subjects and obstructive sleep apnea patients, respectively.

Three stage classification (Wake/REM/NREM) of sleep has been derived using the analyses of spectral components of the heartbeats extracted from multichannel BCG based on EMFi sensors [58]. By applying a hidden Markov model only on BCG, they achieved a total accuracy of $79 \%$ (kappa index of 0.43 ) compared to clinical sleep staging from PSG. The performance was enhanced by combining the time variant-autoregressive model (TVAM) and wavelet discrete transform (WDT) with the quadratic (QD) or linear discriminant (LD) analysis [59]. The QD-TVAM algorithm achieved a total accuracy of 76.8\% (kappa index of 0.55 ), while LD-WDT achieved a total accuracy of $79 \%$ (kappa index of 0.51). Although there was also a study done for sleep stage classification into four levels (wake/REM/deep sleep/light sleep) with ECG [60], four-level sleep stage classification with BCG is not reported yet. With the ECG signal, Tanida et al. classified the sleep stage with HRV analyzed for each 60-s epoch of ECG and calculated at three frequency band powers. Their results for minute-by minute agreement rate ranged from $32 \%$ to $72 \%$ with an average of $56 \%$ for ten healthy women.

Sleep monitoring based on BCG technology has a potential to provide both continuous and longitudinal information on a subjects' sleep quality and may take a role as a predictive screening method prior to the sleep studies based on PSG. It could also fill the gap among PSG of whole night examination and portable ambulatory PSG, which can be applied at home and simplified with, for example, a wrist worn movement sensor.

\section{Chair-Based BCG and SCG systems}

Chair-based systems have mainly used electromechanical film (EMFi) sensors based on piezoelectric transduction. Koivistoinen et al. attached EMFi sensors to a chair to measure BCG signals from two seated subjects, and found the signal shape to be similar to other BCG measurements from the literature
[61]. Walter et al. placed an EMFi mat in the cushion of the driver's seat in a car to measure the BCG for automatically monitoring driver fitness [62]. These systems provide a means for measuring BCG or SCG signals from patients who cannot stand still on their own, minimize motion artifacts, and allow the user to be comfortable during the measurement. The main disadvantages for chair-based BCG recording are the reduction of signal amplitude compared to measurements using table, bed, or weighing scale systems, and the effects of postural changes on signal quality.

\section{Signal PROCESSING AND Modeling}

\section{A. Heartbeat Detection}

Since heart rate is regulated by the autonomic nervous system, the analysis of HRV is currently employed to obtain physiological and clinical information on the level of sympathetic and parasympathetic drive to the heart. Even though ECG is the most widely used biological signal to evaluate heart rate dynamics, BCG may also be used. Due to its easier application for monitoring in contrast to the inconvenience of attaching electrodes to the skin in ECG measurement, BCG may facilitate the assessment of heart rate dynamics in daily life [63].

Heartbeats may be identified by the J-wave peak in the BCG signal, i.e., the point of highest amplitude in the BCG waveform. Heart rate is evaluated by measuring the interval between consecutive J-peaks, the J-J interval. As there are many algorithms to detect the R-peak in ECG, there are also various methods to detect the J-peaks or heart beat from BCG. Since BCG can be measured in different settings with different type of sensors, the peak-detection algorithm should be selected to optimize the performance considering the characteristics of measured BCG. A heartbeat detection algorithm which showed high performance in R-peak detection from ECG can be applied with minor modification for J-peak detection. Generally the peak detection procedure is applied to select the highest value in amplitude as the J-peak within the sliding window after some preprocessing to increase signal-to-noise ratio (SNR) and to reject artifacts due to motion or other interferences.

Choi et al. demonstrated increased detection performance with a dedicated algorithm, which finds local peaks in four divided subintervals within a period and selects the maximum peak as J-peak from these local peaks with some rejection rules [44]. Jansen et al. applied a detection method based on a "template matching" rule by evaluating a correlation function in a local moving window [64], a method which was further refined and developed by Shin et al. [65]. Although this method requires template design in its first stage, Shin et al. successfully applied it to several types of BCG signals acquired from air mattress, load cells, and EMFi sensors. The results showed $95.2 \%$ of sensitivity and $94.8 \%$ of specificity in average for five subjects and three types of BCG signals. Additional methods for heartbeat detection from BCG signals include those which combine different estimators [46], [66], [67], and methods which use wavelets to preprocess the signal prior to peak detection [53], [68].

Heart rate was estimated from the spectral domain specially focusing on third harmonics especially in BCG signals acquired 
with fiber optic sensors [45]. The results showed an error less than $0.34 \mathrm{beat} / \mathrm{min}$ in $2^{\circ} \mathrm{min}$ averaged heart rate. Heartbeat intervals were calculated with the cepstrum method, by applying FFT for short time windows including pair of consequent heart beats [48]. Relative error of the method was $0.35 \%$ for 15 night recordings with six normal subjects after rejecting movement artifacts. Since the results of heart beat detection are not perfect, generally visual editing is required to correct the errors in peak detection for further application like HRV analysis. Multichannel fusion techniques have also been demonstrated recently for BCG-based heartbeat detection [48], [69].

Recently, Paalasmaa et al. [70] and Brueser et al. [71] both verified heartbeat detection algorithms on large datasets containing hundreds of thousands of heartbeats recorded in uncontrolled environments. Paalasmaa et al. used hierarchical clustering to first infer a heartbeat shape from the recordings, then beat-tobeat intervals were found by determining positions at which this template best fit the signal. The mean beat-to-beat interval error was $13 \mathrm{~ms}$ from 46 subjects in the clinic, home, single bed, double bed, and with two sensor types. Brueser et al. demonstrated robust estimation of heartbeats for 33 subjects of which 25 were insomniacs, with a mean beat-to-beat interval error of $0.78 \%$. Their method used three short-time estimators combined using a Bayesian approach to continuously estimate interbeat intervals. Automatic template learning approaches were also presented by Brueser et al. in 2011 with low error [51].

Performance of HRV analysis using BCG measured on weighing scale-type load cell is evaluated in reference to the ECG during the resting and under each condition of Valsalva and postexercise sessions that induce cardiac autonomic rhythm changes [72]. Time domain, frequency domain, and nonlinear domain HRV parameters were evaluated on 15 healthy subjects to assess the cardiac autonomic modulation under each of these conditions. For all subjects and for all experimental sessions, HRV parameters calculated from BCG peak intervals are statistically not different from those obtained from the reference ECG. The results showed high performance with relative errors of 5.0-6.0\% and strong correlation of 0.97-0.98 in average for these three states compared with the results from ECG peaks. The errors were relatively high in HRV parameters reflecting the high-frequency characteristics of heart rates such as $\mathrm{HF}, \mathrm{LF} / \mathrm{HF}$ in the spectral analysis, pNN50 in time-domain analysis, and SD1 in nonlinear analysis. This is considered to be caused by the inaccuracy in detecting peak from the less sharp J-peak of BCG compared to the R-peak in ECG. HRV estimates with BCG have also been compared to the PPG, and the correlation between the two was found to be high [73]. Preliminary work was recently presented by Brueser et al. for unsupervised HRV estimation from BCG signals [74].

\section{B. Noise and Interference Reduction}

Several sources of noise and interference can potentially corrupt BCG and SCG measurements taken using modern systems. These include sensor and circuit noise [75], motion artifacts [15], [21], [76], [77], and floor vibrations (for standing BCG measurements) [78].
Both BCG and SCG represent low-level signals that con- 462 tain very low-frequency information - this can lead to problems 463 with flicker (1/f) noise in the sensor interface circuit corrupt- 464 ing the measurements. Furthermore, many diseased subjects, 465 and elderly subjects, have smaller signal amplitudes compared 466 to the healthy young population [79]. The sensor and circuit 467 noise were characterized and reduced for weighing-scale-based 468 BCG systems using an ac-bridge amplifier approach [75]. This 469 approach led to a SNR improvement of $6 \mathrm{~dB}$.

For ambulatory and standing subjects, motion artifacts present the greatest potential obstacle to achieving reliable measurements. Unlike bed or chair systems, where the subject stays generally still for the measurement, postural sway, or ambulation can create unwanted peaks or distortion in the measured signals. Motion artifact detection for standing BCG measurements was accomplished using auxiliary sensors as noise references; then, gating the BCG signal based on the detection of excessive noise [76], [80]. In one study, the noise reference was an extra strain gauge added to the scale to detect postural sway [76]. In another study, the rms power of the electromyogram signal from the feet, indicating the presence of increased muscle contractions due to excessive movement, was used as a noise gate for the BCG [80]. Pandia et al. presented preliminary methods for cancelling motion artifacts in SCG signals from walking subjects, improving overall heartbeat detection [77]. Di Rienzo et al. used an automatic selection of movement-free data segments from daily recordings of SCG signals from ambulant subjects, followed by an ECG triggered ensemble averaging to reduce signal noise [21]. This enabled, for the first time, the assessment of systolic time interval profiles during normal daily living.

BCG measurements taken in a direction orthogonal to the plane of the floor can potentially be corrupted by floor vibrations - this can particularly pose a challenge for measurements taken on a vehicle [62] or plane [81]. Walter et al. instrumented the seat of a car with an EMFi mat to measure the BCG, aiming to use the information to monitor driver fitness [62]. However, with the engine turned on, the BCG was corrupted by vibration artifacts and rendered unusable. Inan et al. used an auxiliary sensor for vibration detection and adaptive noise cancellation to cancel floor vibration artifacts in the BCG measurement [78]. In this study, high-quality BCG measurements were successfully demonstrated from a subject standing on a bus with the engine turned on and idling. Additionally, it was observed that low-noise SCG waveforms could be obtained in a subject sitting in the metro, while a train was going by, with the above mentioned ensemble averaging approach [21].

\section{Signal Modeling}

Modeling of SCG and BCG provides a tool to better un- 509 derstand the genesis of waves in these signals and to simulate 510 their morphological changes with different myocardial abnor- 511 malities. Modeling of BCG goes back to the early years of 512 ballistocardiographic research [79].

In most BCG recording systems, the recording device is quite 514 small compared to the human body and the platform on which 515 it rests. It is also far away from the heart in most cases; thus, 516 


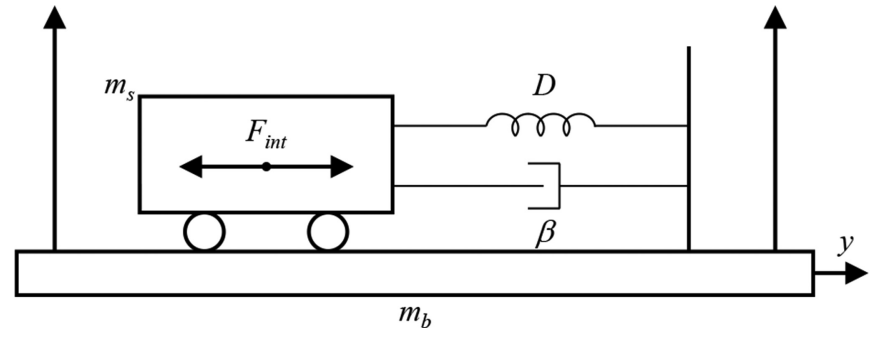

Fig. 3. Schematic showing the subject (with mass, $m_{s}$ ) and the BCG recording system (with mass, $m_{b}$ ) coupled by a spring dashpot system.

TABLE II

DESCRIPTIONS OF VARIABLES FOR SIGNAL MODELING

\begin{tabular}{lc}
\hline \hline Variable & Description \\
\hline$F_{\text {int }}$ & Internal forces \\
$\beta$ & Damping constant \\
$y$ & Displacement or (in subscript) indicating \\
$\dot{y}$ & head-to-foot direction \\
$\ddot{y}$ & Velocity \\
$D$ & Acceleration \\
$m_{s}$ & Spring constant \\
$m_{b}$ & Mass of subject \\
\hline \hline
\end{tabular}

the volume of the heart has been neglected in such models. The heart has been modeled like a point source providing the flow to the circulation system model [82]. Such a model is in accordance with the classical definition of BCG to be resulted through movement of center of gravity of the body and platform. On the contrary, in SCG the recording device (i.e., accelerometer) is near the heart and the volume of the heart cannot be neglected in any model dealing with SCG or any other precordial vibration signal. Thus, except for some preliminary efforts [83] SCG modeling has not been pursued by many researchers, probably because of the complications associated with such a model.

In ballistocardiographic research, one can study the events within human body that cause its movement in space, regardless of the recording device or to study the properties of instruments recording them and how their record relates to the movement originating them. Both of these two approaches are briefly introduced.

1) Modeling the Recording Device: During the early years of ballistocardiographic research, several different instruments were used to measure BCGs, from beds hanging from the ceiling [84] to tables strongly coupled to ground [1]. These instruments were giving different records from the same normal subjects. So, efforts were made to model the effect of these instruments on BCG morphology. Limiting ourselves to the head-foot direction the equation giving the components along the $y$-axis (Fig. 3, variables defined in Table II) reads:

$$
\left(F_{\text {int }}\right)_{y}-\beta \dot{y}-D y=\left(m_{s}+m_{b}\right) \ddot{y} .
$$

After sorting and substituting $\left(F_{\text {int }}\right)_{y}$ into $m_{s} \ddot{y}_{c}$ (where $\ddot{y}_{c}$ is the acceleration of center of mass of body):

$$
\left(m_{s}+m_{b}\right) \ddot{y}+\beta \dot{y}+D y=m_{s} \ddot{y}_{c} .
$$

From the above equation, three different classic types of 545 BCGs can be conceived based on the fact that which terms on the left side of the above equation can be neglected. The first is

$$
\left(m_{s}+m_{b}\right) \ddot{y}=m_{s} \ddot{y}_{c}
$$

which means that the movement of bed and body is proportional 548 to the movement of the center of gravity. A good approximation 549 of this special case is when the ballistocardiograph is weakly 550 coupled to the environment such as ultralow frequency BCG (ULF-BCG) systems.

The second type is when:

$$
\dot{y}=\frac{m_{s}}{\beta} \ddot{y}_{c}
$$

which represents Nickersons's low-frequency (LF) BCG and 554 the third type is when:

$$
y=\frac{m_{s}+m_{b}}{\beta} \ddot{y}_{c}
$$

which refers to the situation when BCG is strongly coupled to its environment, which were categorized under high-frequency BCG (HF-BCG). In other words, when the resonance frequency of the BCG platform is much higher than heart frequency, then its displacement is proportional to the internal acceleration of body's center of gravity.

From this theoretical evaluation, it is clear that very different results will be obtained when one records any one aspect of motion such as displacement or acceleration from each of the three ideal types of ballistocardiographs [82]. However, there is a fourth category of classical BCGs, which are the direct body recordings based on AHA consensus paper on BCG terminology [85]. Direct body BCGs were always criticized for their inconsistencies [82].

2) Modeling the Internal Forces: Starr started on BCG modeling, where arteries were segmented into $3-\mathrm{cm}$ long pieces and mass of blood in the aortic segment closest to the aortic valve was multiplied by acceleration, derived from cardiac ejection curve, to calculate force. This was repeated when the blood volume shifted to the next segment [82].

A more comprehensive model of human systemic arterial tree with distributed properties was constructed in early 1960s by Starr and Noordergraaf [82] and was improved later on by Westerhof et al. [86]. This model was based on the fact that, when using ULF systems, in which the body was free to move in space in the head-foot axis, it was observed that the body moved first footward and then headward during the cardiac cycle. This was explained as a movement to counteract the displacement of the blood mass, that, shortly after the onset of systole, is first driven headward out of the heart to distend the great vessels, and later footward, as the pulse wave spreads peripherally and blood accumulates at a great distance from the heart in the more peripheral vessels.

The model divided the arterial tree in 115 segments and calculated the position of the body's center of gravity in the longitudinal direction $y_{c}(t)$, as a function of time, by numerical integration of the products of the excess masses of each segment during the interval $t$, and the distance $y_{i}$ between the centre of 
each segment and the reference plane. Noordergraaf's model was successful in quantitatively predicting the amplitudes of ULF BCG waves and in giving an explanation for the origin of the main peaks. The model was verified on the data acquired from an astronaut in MIR station [87], where by using the longitudinal BCG recorded in space the model could be used to derive the aortic flow.

\section{Human SubJects Studies With Modern Systems}

\section{A. Correlation Studies With Healthy Subjects}

Originally, BCG and SCG were proposed as diagnostic tools for the clinic - for example, a patient would lie on a Starr BCG table, the recording would be printed on a strip chart, and the physician would read the recording to make a diagnosis regarding the patient's cardiovascular health [1], [5]. However, the large intersubject variability in the signals hampered this approach, particularly given the limited tools available at that time for signal analysis. On the contrary, studies have shown that the intrasubject variability in the signals over serial measurements is actually low [15] — except in the presence of changing cardiovascular health. For this reason, in the past decade the BCG and SCG have been proposed as tools for monitoring changes in the same patient's health overtime. Then, the subject is his/her own control, and intersubject variability is no longer an obstacle.

To uncover the clinical relevance of BCG and SCG signal features, and to pave the way for future studies with clinical populations, several researchers conducted human subjects studies with a healthy population using modern instrumentation and analysis tools. These studies were mainly designed with a noninvasive protocol for altering the hemodynamics and timing intervals of the heart—such as exercise, Valsalva maneuver, whole-body tilt testing, or lower body negative pressure (LBNP) - then, comparing the changes in the BCG or SCG waveform to changes in a reference standard measurement, such as impedance cardiography (ICG) or Doppler ultrasound.

For both BCG and SCG signals the amplitude (or rms power) components have been shown to modulate with changes in left ventricular function - in particular, changes in stroke volume (SV) or cardiac output (CO). Castiglioni et al. measured clavicular SCG signals before and immediately after exercise and compared the percent changes in the peak-to-peak amplitude of the SCG to changes in $\mathrm{CO}$ as measured by the finometer model flow method, finding a strong correlation for four data points taken from four subjects [24]. Inan et al. further demonstrated that the changes in rms power resulting from exercise, measured during $10 \mathrm{~min}$ of recovery time, were strongly correlated to changes in CO measured by Doppler ultrasound for 275 data points taken from nine subjects [88]. Tavakolian etal. trained a neural network to estimate SV from SCG parameters and tested this classifier on a separate testing dataset, finding an average correlation coefficient of 0.61 , and Bland-Altman agreement limits $(95 \%$ confidence) of $+7.4 \mathrm{~mL},-7.6 \mathrm{~mL}$ for 4900 heartbeats analyzed from eight participants [16]. It is important to note that these error bands are larger than what would be needed for absolute volume estimation using the SCG; however, this may be of interest for future research.
Many researchers have also examined the time intervals both within the signals themselves, and between BCG / SCG signal features and other physiological measurements (e.g., ECG or PPG), to form a relationship between these timing intervals to more well-known parameters [e.g., preejection period (PEP), pulse transit time (PTT), or left ventricular ejection time (LVET)]. The time interval between the ECG R-wave peak and the BCG J-wave peak has been proposed as a surrogate for the PEP - a measure of the IVC period of the heart and an index of cardiac contractility [30], [89]. These authors used the Valsalva maneuver and/or whole body tilt testing to modulate the PEP by changing the autonomic balance between parasympathetic and sympathetic drive, and compared the R-J interval to the PEP measured using ICG. Etemadi et al. demonstrated a strong correlation $\left(R^{2}=0.86\right)$ between the R-J interval and the PEP for 2126 heartbeats across ten subjects performing the Valsalva maneuver [89]. He et al. showed similar results for one example subject with both the Valsalva maneuver and whole-body tilt testing [30]. Tavakolian etal. proposed the interval between the ECG Q-wave and the SCG AO-point as a surrogate for PEP, and found strong correlation between this interval and PEP measurement using ICG and Doppler ultrasound in 25 subjects [16].

Researchers have also attempted to extract data from the BCG relating to blood pressure (BP), leveraging the known relationship between pulse wave velocity estimated using PTT, and Pinheiro et al. suggested the use of BCG and PPG for PTT estimation [90]. Shin et al. compared the R-J interval of the BCG, modulated using the Valsalva maneuver, to beat-by-beat systolic BP (SBP) measurements taken using the Finapres system, finding a strong correlation [39]. Nevertheless, Casanella et al. found that, in case of hemodynamic changes induced by paced respiration, this correlation between R-J interval and SBP was dependent on the subject and was not always observed [91]. Winokur et al. found, for one example subject, that the time interval between the BCG and the PPG signal, both measured at the ear, were correlated to PTT, and could thus be used to estimate BP [31].

Another important interval is the duration of systolic ejection, the LVET, as it provides an indication of what percentage of the cardiac cycle is being devoted to ejection compared to filling. Tavakolian et al. used LBNP to simulate hemorrhage, and found that LVET measurements taken using SCG were significantly different at various stages of LBNP, and correlates with the LBNP levels $(R=0.90)$ for 32 subjects [92]. Di Rienzo et al. found that with exercise LVET changes measured using wearable SCG are in line with the changes reported in the literature and obtained by traditional laboratory techniques [21], [93].

\section{B. Clinical Findings From Patients With Cardiovascular Disease}

Modern ballistocardiography and seismocardiography sys- 698 tems may be capable of monitoring slow, longitudinal changes 699 in cardiac function associated with a number of cardiovascu- 700 lar diseases. Timely noninvasive detection of subtle changes in 701 cardiac pathophysiology may one day enable daily drug dosage 702 adjustments, thus reducing costly and morbid rehospitalizations 703

\section{9} 650 651 652 653 654 655 656 657 658 659 660 661 662

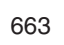

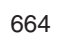
(6) 666 667 668 669 670 671 672 673 674 675 676 677 8

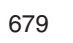
680 681 682

\section{3} 684 685

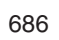

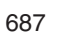

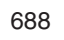

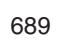
$\infty$

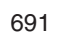

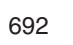

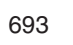

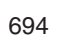
695 s. 
[94]. At this moment, the feasibility of this approach is investigated by the ongoing LAPTOP-HF study which, however, uses an implantable right atrial pressure sensor coupled to a mobile device that allows daily automatic dosage adjustment [95].

Fortunately, the basis for the SCG's clinical utility was begun in 1990 with the initial use of high sensitivity, LF accelerometers to measure precordial vibrations [96]. Significant features of the SCG waveform were identified and associated with key events in the cardiac cycle [17]. This allowed the accurate measurement of these features (e.g., ACs and MOs) using one sensor, greatly simplifying the calculation of CTIs.

A large body of work exists on the utility and efficacy of CTIs [97], [98]. This knowledge combined with the ability to make accurate, repeatable quantitative measurements using the SCG resulted in the ability to conduct clinically relavent crosssectional studies. Subsequently, clinical studies were undertaken to determine if the SCG could be used to identify changes in the SCG waveform resulting from myocardial ischemia [99].

The SCG's clinical utility in enhancing the diagnostic outcome of a graded exercise stress test was first shown in [100]. A large multicenter study demonstrated that when the combined results of the ECG and SCG were used, the predictive accuracy of detecting physiologically significant coronary artery disease was increased significantly over the results of the ECG alone [7].

The introduction in the early 1990s of lightweight $(<25 \mathrm{~g})$ accelerometers, whose working range extended below $1 \mathrm{~Hz}$, made possible other clinical settings for the SCG. The SCG as a magnetic-field-compatible alternative to the electrocardiogram for cardiac stress monitoring [101] was made possible using a newly introduced light weight piezoelectric accelerometer (336C, PCB Piezotronics, Depew, NY, USA).

The SCG was used to measure CTI's during atrial, ventricular, and biventricular pacing, as compared to normals [102]. One of the studies objectives was to determine the utility of the SCG in cardiac resynchronization therapy (CRT). This study was the first to use 3 SCG traces for analysis, i.e., one accelerometer was placed on the xyphoid process, a second over the apex at the fourth intercostal, and a third on the right carotid pulse.

In 1994, the SCG was used to make accurate longitudinal measurements in a study of the effects of elgodiphine on cardiac hemodynamics [103]. In a sports medicine application, exercise capacity was evaluated using the SCG [104]. A more extensive review of the SCG is available in [105].

As a note of interest, the combined patient population of the myocardial ischemia studies [7], [100] is close to 2000 and consists of both healthy and disease subjects. All the raw data were recorded with the same instrumentation (SCG 2000, SeisMed Instruments, Minneapolis, MN, USA) associated with these datasets are complete patient demographics. A project is underway to make the raw data available on the PhysioNet website for study by interested researchers [105].

More recent findings with BCG and SCG further support that the signals have great potential in allowing proactive cardiac disease management without a costly implantable device. However, despite stated clinical and/or physiologic motivations, the overwhelming majority of modern BCG/SCG findings continue to be from healthy subjects [106]-[108]. Notable exceptions in- clude a bed-mounted BCG system for automated detection of 761 atrial fibrillation [109], the observation of reduced signal ampli- 762 tude in the setting of premature atrial or ventricular contractions 763 [15], and the reduction of signal consistency in heart failure 764 patients concordant with worsening clinical outcome [110]. $\quad 765$

One particular subset of patients is particularly well suited for 766 study using cardiomechanical signals, those undergoing CRT. 767 CRT patients have abnormal cardiac conduction causing in a 768 significant delay between the pumping action of the various 769 chambers of the heart. CRT involves precisely adjusting the 770 timing of a multichamber pacemaker to reduce or remove these 771 delays. Such timing is difficult to ascertain using available tech- 772 nologies, spawning the field of "CRT optimization." Researchers 773 recently demonstrated the benefits of intracardiac acceleration 774 monitoring in performing CRT optimization [111], a finding 775 preliminarily corroborated by BCG findings as well [8].

\section{3-D Ballistocardiography and Microgravity Studies}

As the sections on instrumentation earlier in this review have indicated, measurements of BCG (in particular) are constrained by the coupling of the body to the ground, a direct result of the influence of gravity. As such, full 3-D recordings of the BCG are difficult in the terrestrial environment, and much of the focus has been on accelerations in the coronal plane (the $X Y$ plane as defined in the section on measurement axes).

Given this limitation, it is therefore not surprising that the idea of measuring the BCG in a subject in free-fall (weightlessness, zero-G, microgravity) was an obvious target of investigation. The first such experiment was performed in the 1960s in parabolic flight, with the subject strapped into a "tub," which was itself instrumented to record the BCG [9]. Despite the limited periods of microgravity available (typically $\sim 20 \mathrm{~s}$ ) and the subject restraints, recordings of good quality were obtained.

Spaceflight represents the other obvious environment in which the "true" 3-D BCG can be recorded. The earliest recordings were made by the Soviets on Saluyt-6 [10] and consisted of a series of five recordings were performed in two crew members of a long duration mission on days 46,71, 98, 133, and 175 . A piezoelectric sensor, attached close to the center of mass, recorded ballistic forces in the feet-to-head axis during breath holding experiments. Individual changes were seen during the mission with maximum amplitude of the IJ wave occurring on day 133. Measurements were also made during the Spacelab-1 mission aboard the Space Shuttle in 1983 [112]. These experiments were conducted in two subjects at two occasions during this short duration spaceflight and showed an increase of the overall systolic accelerations along the longitudinal axis in microgravity.

Perhaps the best-analyzed dataset of the BCG in spaceflight came from measurements made during the Spacelab D-2 mission in 1993. During that flight, extra time became available (due to an extension of the overall mission length), and an experiment was hastily conceived, approved, implemented, and performed to measure 3-D BCG in a free-floating subject. Parenthetically, this may be one of the fastest spaceflight experiments ever developed with the time from concept, to collection of the data 


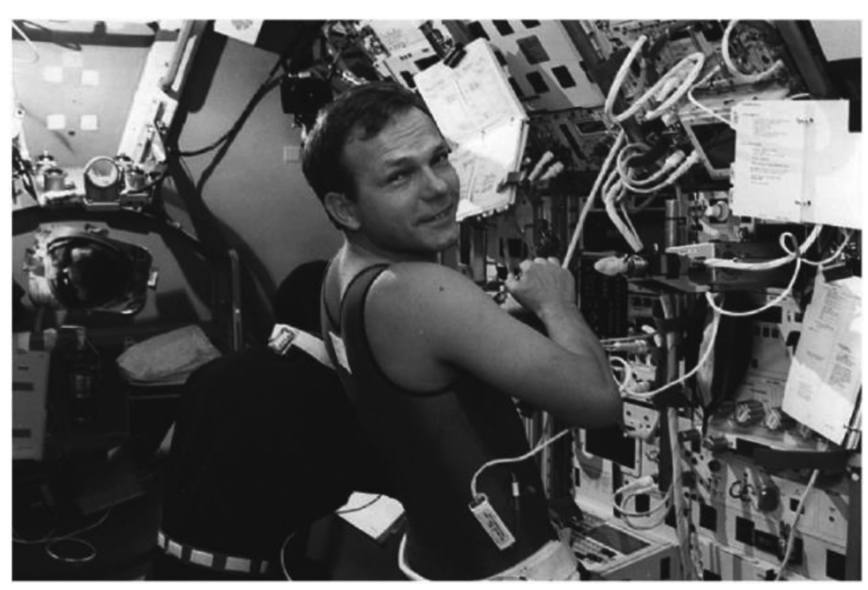

Fig. 4. Subject in D-2 shown wearing the snuggly-fitting suit incorporating a respiratory inductance plethysmograph and ECG. Photo Credit: NASA.

(including approval of an institutional review board) was only 4-5 days, surely some sort of record. The experiment utilized data from a free-floating subject instrumented with an ECG and wearing a snuggly fitting suit that measured respiratory motion using an impedance plethysmograph (see Fig. 4). This instrumentation was a part of the Anthrorack series of human studies managed by the European Space Agency. The second cruicial piece of instrumentation was a set of high-fidelity triaxial accelerometer that were attached to the vehicle and used for measuring the accelerations imparted by crew activity in the Spacelab. The sensor package was detached from the vehicle and taped to the lumbar region of the subject, near to the (presumed) center of mass. Data were then recorded as the subject remained stationary and free floated in the center of the Spacelab, providing a continuous recording, free of interruptions of $146 \mathrm{~s}$. In order to synchronize the two separate data streams, collisions with the Spacelab structure, which disrupted signals in both data streams, were used as posthoc event source [11].

The data from the D-2 study and some subsequent studies provided valuable insight into several aspects of the BCG. In particular there were four major conclusions derived from this dataset.

1) Lung volume greatly influences the accelerations recorded, especially in the longitudinal (head-to-foot) body axis (see Fig. 5), with the implication being that there is better coupling between the heart and the body in the longitudinal axis at higher lung volumes [11]. Interestingly, the actual direction of respiratory motion (mid inspiration versus mid expiration) had only minimal influence of the BCG.

2) Data derived from short periods of microgravity in parabolic flight are largely equivalent to data obtained in sustained microgravity [113].

3) The BCG has a plane of symmetry that is primarily sagittal. This suggests that $2-\mathrm{D}$ recordings performed in a supine subject (i.e., coronal recordings) fail to capture a significant portion of the effect of the blood ejection on the body, complicating their interpretation [113].
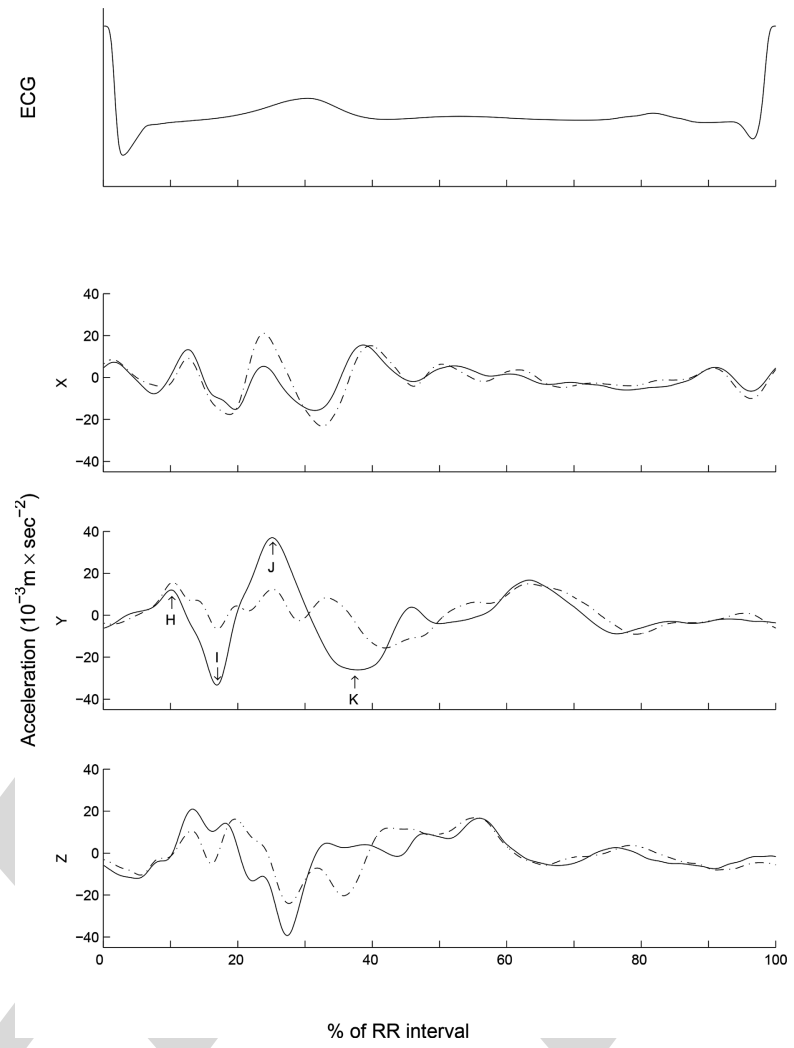

Fig. 5. The 3-D BCG recorded in spaceflight in a free-floating subject, at the end of a normal expiration (dashed lines, functional residual capacity, FRC), and at the end of a normal inspiration (solid lines, FRC + tidal volume). From [11].

4) The accelerations that are recording in a 2-D system are 855 only modestly correlated with the true 3-D accelerations 856 that actually occur, again complicating their interpretation 857 [113].

BCG flight experiments were also an integral part of the 859 Russian cardiovascular research program for the orbital sta- 860 tion MIR. BCG along the head-to-foot direction was measured 861 in three crew members during the second MIR mission in 862 1988 and compared to SCG recordings. Significant changes 863 of the BCG amplitudes (HI, IJ, JK) during the long-term flight 864 were described together with large inter individual differences. 865 The first true 3-D-BCG recordings were made during the sixth 866 MIR mission in 1990 in two crew members on flight days 56867 and 110. Three new piezoelectric sensors were used placed 868 in perpendicular planes in a small cylindrical box with a di- 869 ameter of $40 \mathrm{~mm}$ and a height of $20 \mathrm{~mm}$. The sensitivity of 870 the sensor was $20 \mathrm{mV} / \mathrm{m} / \mathrm{s}^{2}$. The sensor was placed between 871 the scapulae using rubber belts and a metallic plate. The spe- 872 cial amplifier (BCG-3) was connected to the recording unit 873 "Gamma-1," and the data were transmitted telemetrically to 874 the ground station. In summary, no dramatic changes in the vec- 875 tor sum were detected. Maximum forces ranged from 5.85 to 876 10.18 N. However, profound individual changes of the shape, 877 amplitude, and timing of the BCG, especially in the lateral 878 and dorso-ventral plane have been found. Finally, combined 879 BCG and SCG measurements have been made every month 880 in space during the 14 months space flight of Valeri Poljakov, 881 
15th to 17th MIR missions (Russian-Austrian flight experiment "Pulstrans") [114].

\section{STANDARDS AND OPEN ISSUES}

\section{A. Need For a Standardization}

From the analysis of the literature, it appears that important methodological aspects concerning BCG and SCG analysis are still characterized by a certain level of ambiguity. These include

1) Definitions of BCG and SCG Signals: In the literature, the definition of BCG and SCG is not univocal and the "BCG" term is even sometimes used for SCG signals.

2) Nomenclature: Since BCG and SCG waveforms are mostly different (although they might have some common features to be investigated) it is reasonable to use a specific nomenclature for defining peaks and valleys of each signal. The prevalent annotation for BCG was proposed by Starr et al. [1], for SCG by Crow et al. [17]. However, there are some disagreements on these annotations, and in some instances, SCG peaks are termed with the BCG annotation.

3) Indication of Site of Measurement, Characteristics of sensor, Sensor Axis Orientation: These pieces of information are crucial for data comparison and interpretation, but unfortunately are not invariably reported in scientific communications.

A standardization or at least a common position on the above issues would greatly facilitate the understanding and comparison of published results, the exchange of data, and the design of new experimental protocols in this area.

\section{B. Open Issues}

A number of open issues remain to be addressed in this field to improve the understanding and applicability of BCG and SCG signals. Hereafter, we provide just a short list of these issues.

1) The biological meaning of BCG and SCG deflections not yet annotated and their clinical relevance.

2) Possible common features of the BCG and SCG signals.

3) Further parameters derivable from the analysis of the BCG and SCG 3-D vectors.

4) Effects of respiration, posture, right ventricle, and sensor adherence on the signal waveform/quality.

5) How to facilitate the use of these signals in clinical practice?

6) Reference values for healthy and diseased subjects for both types of signals, and for a wide range of body types/sizes, and ages.

\section{CONCLUSION AND AREAS FOR FUTURE INVESTIGATION}

The recent advances in the BCG and SCG field indicate the strong potential of these measurements to address wide variety of clinical needs, in particular monitoring or trending the cardiomechanical health of patients outside of the clinic. Both BCG and SCG measurements can be taken using inexpensive and unobtrusive sensors, making them ideally suited, for example, for home monitoring of chronic diseases. Nevertheless, to maximize our ability to interpret these signals, the physiological origins of both signals must be studied further and elucidated. Furthermore, there is a need to be able to map each measurement modality to another using cardiovascular and mechanical modeling of the body, such that any BCG or SCG waveform amplitude, timing, or morphology measured using one modality can be translated quantitatively to another. For example, if a bed-based recording in the dorso-ventral axis yielded a peak BCG J-wave amplitude of $2 \mathrm{~N}$, system modeling tools are needed to compare this to a corresponding J-wave amplitude measured using a weighing scale. Finally, an extensive, open database of BCG and SCG signals, processing tools, and even microprocessor code needs to be made available to massively expand the capability of researchers around the world to investigate these signals, use them in their own settings, and grow the field from a niche into an established technique, routinely used in clinical practice.

\section{REFERENCES}

[1] I. Starr, A. J. Rawson, H. A. Schroeder et al., "Studies on the estimation of cardiac output in man, and of abnormalities in cardiac function, from the heart's recoil and the blood's impacts; the ballistocardiogram," Amer. J. Physiol., vol. 127, pp. 1-28, 1939.

[2] B. S. Bozhenko, "Seismocardiography-a new method in the study of functional conditions of the heart [Article in Russian]," Ter Arkh, vol. 33, pp. 55-64, 1961.

[3] J. W. Gordon, "Certain molar movements of the human body produced by the circulation of the blood," J. Anat. Physiol, vol. 11, pp. 533-536, 1877.

[4] D. M. Salerno and J. Zanetti, "Seismocardiography for monitoring changes in left ventricular function during ischemia," Chest J., vol. 100 pp. 991-993, 1991 .

[5] I. Starr and F. C. Wood, "Studies with the ballistocardiograph in acute cardiac infarction and chronic angina pectoris," Amer. Heart J., vol. 25, pp. 81-101, 1943.

[6] H. Mandelbaum and R. A. Mandelbaum, "Studies utilizing the portable electromagnetic ballistocardiograph: IV. The clinical significance of serial ballistocardiograms following acute myocardial infarction," Circulation, vol. 7, pp. 910-915, 1953.

[7] R. A. Wilson, V. S. Bamrah, J. J. Lindsay et al., "Diagnostic accuracy of seismocardiography compared with electrocardiography for the anatomic and physiologic diagnosis of coronary artery disease during exercise testing," Amer. J. Cardiol., vol. 71, pp. 536-545, 1993.

[8] L. Giovangrandi, O. T. Inan, R. M. Wiard et al., "Ballistocardiography: A method worth revisiting," in Proc. IEEE Annu. Int. Conf. Eng. Med. Biol. Soc., 2011, pp. 4279-4282.

[9] W. C. Hixson and D. E. Beischer, "Biotelemetry of the triaxial ballistocardiogram and electrocardiogram in a weightless environment," Naval School Aviat. Med., Pensacola, FL, USA, 1964.

[10] R. M. Baevsky and I. I. Funtova, "Ballistocardiographic examinations of the salyut-6 fourth expedition crew members," Komsich Biologiya Aviak Medit, vol. 16, pp. 34-37, 1982.

[11] G. K. Prisk, S. Verhaeghe, D. Padeken et al., "Three-dimensional ballistocardiography and respiratory motion in sustained microgravity," Aviat. Space Environ. Med., vol. 72, pp. 1067-1074, 2001.

[12] P. F. Migeotte, S. De Ridder, J. Tank et al. " "Three dimensional ballistoand seismo-cardiography: HIJ wave amplitudes are poorly correlated to maximal systolic force vector," in Proc. IEEE Annu. Int. Conf. Eng. Med. Biol. Soc., 2012, pp. 5046-5049.

[13] R. M. Wiard, H. J. Kim, C. A. Figueroa et al., "Estimation of central aortic forces in the ballistocardiogram under rest and exercise conditions," in Proc. IEEE Annu. Int. Conf. Eng. Med. Biol. Soc., 2009, pp. 2831-2834.

[14] M. Di Rienzo, P. Meriggi, F. Rizzo et al., "A wearable system for the seismocardiogram assessment in daily life conditions," in Proc. IEEE Annu. Int. Conf. Eng. Med, Biol. Soc., 2011, pp. 4263-4266.

[15] O. T. Inan, M. Etemadi, R. M. Wiard et al., "Robust ballistocardiogram acquisition for home monitoring," Physiol. Meas., vol. 30, pp. 169-185, 2009. 
[16] K. Tavakolian, "Characterization and analysis of seismocardiogram for estimation of hemodynamic parameters," Ph.D. dissertation, Dept. Appl. Sci, School Eng. Sci., Simon Fraser Univ., Burnaby, BC, Canada, 2010.

[17] R. S. Crow, P. Hannan, D. Jacobs et al., "Relationship between seismocardiogram and echocardiogram for the events in the cardiac cycle," Amer. J. Noninvasive Cardiol, vol. 8, pp. 39-46, 1994.

18] O. T. Inan, M. Etemadi, R. M. Wiard et al., "Novel methods for estimating the ballistocardiogram signal using a simultaneously acquired electrocardiogram," in Proc. IEEE Annu. Int. Conf. Eng. Med. Biol. Soc., 2009 , pp. 5334-5347.

[19] K. Pandia, O. T. Inan, G. T. A. Kovacs et al., "Extracting respiratory information from seismocardiogram signals acquired on the chest using a miniature accelerometer," Physiol. Meas, vol. 33, pp. 1643-1660, 2012.

[20] Y. Chuo and B. Kaminska, "Sensor layer of a multiparameter single-point integrated system," IEEE Trans. Biomed. Circuits Syst., vol. 3, no. 4, pp. 229-240, Aug. 2009.

[21] M. D. Rienzo, E. Vaini, P. Castiglioni et al., "Wearable seismocardiography: Towards a beat-by-beat assessment of cardiac mechanics in ambulant subjects," Autonomic Neurosci., vol. 178, pp. 50-59, 2013.

[22] B. Ngai, K. Tavakolian, A. Akhbardeh et al., "Comparative analysis of seismocardiogram waves with the ultra-low frequency ballistocardiogram," in Proc. IEEE Annu. Int. Conf. Eng. Med. Biol. Soc., 2009, pp. 2851-2854.

[23] M. J. T. Paukkunen, M. T. Linnavuo, and R. E. Sepponen, "A portable measurement system for the superior-inferior axis of the seismocardiogram," J. Bioeng. Biomed. Sci., vol. 3, 2013.

[24] P. Castiglioni, A. Faini, G. Parati et al., "Wearable seismocardiography," in Proc. IEEE 29th Annu. Int. Conf. Eng. Med. Biol. Soc., 2007, pp. 3954 3957.

[25] Y. Chuo, M. Marzencki, B. Hung et al., "Mechanically flexible wireless multisensor platform for human physical activity and vitals monitoring," IEEE Trans. Biomed. Circuits Syst., vol. 4, no. 5, pp. 281-294, Oct. 2010.

[26] R. M. Baevsky, I. I. Funtova, A. Diedrich et al., "Autonomic function testing aboard the ISS using 'PNEUMOCARD'," Acta Astronautica, vol. 65, pp. 930-932, 2009.

[27] P. F. Migeotte, Q. Deliere, J. Tank et al., "3D-ballistocardiography in microgravity: Comparison with ground based recordings," presented at the IEEE Eng. Med. Biol. Soc., Osaka, Japan, 2013, pp. 7012-7016.

[28] E. Luchitskaya, Q. Deliere, A. Diedrich et al., "Timing and source of the maximum of the transthoracic impedance cardiogram (dZ/dt) in relation to the H-I-J complex of the longitudinal ballistocardiogram under gravity and microgravity conditions," presented at the IEEE Eng. Med. Biol. Soc., Osaka, Japan, 2013, pp. 7294-7297.

[29] Q. Deliere, P. F. Migeotte, X. Neyt et al., "Cardiovascular changes in parabolic flights assessed by ballistocardiography," presented at the IEEE Eng. Med. Biol. Soc., Osaka, Japan, 2013, pp. 3801-3804.

[30] D. D. He, E. S. Winokur, and C. G. Sodini, "A continuous, wearable, and wireless heart monitor using head ballistocardiogram (BCG) and head electrocardiogram (ECG)," in Proc. IEEE Аnпи. Int. Conf. Eng. Med. Biol. Soc., 2011, pp. 4729-4732.

[31] E. S. Winokur, D. D. He, and C. G. Sodini, "A wearable vital signs monitor at the ear for continuous heart rate and pulse transit time measurements," in Proc. IEEE Annu. Int. Conf. Eng. Med. Biol. Soc., 2012, pp. 2724-2727.

[32] E. Hyun, S. Noh, C. Yoon et al., "Patch type integrated sensor system for measuring electrical and mechanical cardiac activities," presented at the IEEE Sensors Applications Symposium (SAS), Queenstown, New Zealand, 2014.

[33] G. Balakrishnan, F. Durand, and J. Guttag, "Detecting pulse from head motions in video," in Proc. IEEE Conf. Comput. Vis. Pattern Recog., 2013, pp. 3430-3437.

[34] J. Williams. 1990, Bridge Circuits: Marrying Gain and Balance. Linear Technology Application Note 43.

[35] S. I. Chaudhry, J. A. Mattera, J. P. Curtis, et al., "Telemonitoring in patients with heart failure," N. Engl. J. Med., vol. 363, pp. 2301-2309, 2010.

[36] S. I. Chaudhry, Y. Wang, J. Concato et al., "Patterns of weight change preceding hospitalization for heart failure," Circulation, vol. 116, pp. 1549-1554, 2007.

[37] J. Gomez-Clapers, A. Serra-Rocamora, R. Casanella et al., "Uncertainty factors in time-interval measurements in ballistocardiography," in Proc. 19th IMEKO TC-4 Symp. 17th IWADC Workshop Adv. Instrum. Sens. Interoperability, Barcelona, Spain, 2013, pp. 395-399.

[38] R. Gonzalez-Landaeta, O. Casas, and R. Pallas-Areny, "Heart rate detection from an electronic weighing scale," Phys. Meas., vol. 29, pp. 979-988, 2008.
[39] J. H. Shin, K. M. Lee, and K. S. Park, "Non-constrained monitoring 1075 of systolic blood pressure on a weighing scale," Phys. Meas., vol. 30, 1076 pp. 679-693, 2009

[40] J. Alihanka, K. Vaahtoranta, and I. Saarikivi, "A new method for long- 1078 term monitoring of the ballistocardiogram, heart rate, and respiration," 1079 Amer. J. Physiol., vol. 240, pp. R384-R392, 1981.

1080

[41] Y. Chee, J. Han, and K. S. Park, "Air mattress sensor system with bal- 1081 ancing tube for unconstrained measurement of respiration and heart beat 1082 movements," Phys. Meas., vol. 26, pp. 413-422, 2005.

[42] D. C. Mack, J. T. Patrie, P. M. Suratt et al., "Development and preliminary 1084 validation of heart rate and breathing rate detection using a passive, 1085 ballistocardiography-based sleep monitoring system," IEEE Trans Inf. 1086 Technol. Biomed., vol. 13, no. 1, pp. 111-120, Jan. 2009.

[43] A. Vehkaoja, S. Rajala, P. Kumpulainen et al., "Correlation approach for 1088 the detection of the heartbeat intervals using force sensors placed under 1089 the bed posts," J. Med. Eng. Technol., vol. 37, pp. 327-333, 2013.

[44] B. H. Choi, G. S. Chung, J.-S. Lee et al., "Slow-wave sleep estimation 1091 on a load cell installed bed: A non-constrained method," Phys. Meas., 1092 vol. 30, pp. 1163-1170, 2009.

1093

[45] Y. Zhu, H. Zhang, M. Jayachandran et al., "Ballistocardiography with 1094 fiber optic sensor in headrest position: A feasibility study and a new 1095 processing algorithm," in Proc. 35th IEEE Annu. Int. Conf. Eng. Med. 1096 Biol. Soc., 2013, pp. 5203-5206.

[46] S. Sprager and D. Zazula, "Heartbeat and respiration detection from 1098 optical interferometric signals by using a multimethod approach," IEEE 1099 Trans. Biomed. Eng., vol. 59, no. 10, pp. 2922-9, Oct. 2012.

[47] L. Dziuda and F. Skibniewski, "Monitoring respiration and cardiac activ- 1101 ity using fiber Bragg grating-based sensor," IEEE Trans. Biomed. Eng., 1102 vol. 59, no. 7, pp. 1934-42, Jul. 2012.

[48] J. M. Kortelainen and J. Virkkala, "FFT averaging of multichannel BCG 1104 signals from bed mattress sensor to improve estimation of heart beat 1105 interval," in Proc. IEEE 29th Annu. Int. Conf. Eng. Med. Biol. Soc., 1106 2007, pp. 6685-6688. 1107

[49] J. Paalasmaa, M. Waris, H. Toivonen et al., "Unobtrusive online moni- 1108 toring of sleep at home," in Proc. IEEE Annu. Int. Conf. Eng. Med. Biol. 1109 Soc., 2012, pp. 3784-3788.

[50] F. Wang, M. Tanaka, and S. Chonan, "Development of a PVDF piezopoly- 1111 mer sensor for unconstrained in-sleep cardiorespiratory monitoring," 1112 J. Intell. Mater. Syst. Struct., vol. 14, pp. 185-190, 2003.

1113

[51] C. Bruser, K. Stadlthanner, S. De Waele et al., "Adaptive beat-to-beat 1114 heart rate estimation in ballistocardiograms," IEEE Trans Inf. Technol. 1115 Biomed, vol. 15, no. 5, pp. 778-786, Sep. 2011.

[52] K. Watanabe, T. Watanabe, H. Watanabe et al., "Noninvasive measure- 1117 ment of heartbeat, respiration, snoring and body movements of a subject 1118 in bed via a pneumatic method," IEEE Trans. Biomed. Eng., vol. 52, 1119 no. 12, pp. 2100-07, Dec. 2005.

1120

[53] X. Zhu, W. Chen, T. Nemoto et al., "Real-time monitoring of respiration 1121 rhythm and pulse rate during sleep," IEEE Trans. Biomed. Eng., vol. 53, 1122 no. 12 , pp. 2553-63, Dec. 2006.

[54] J. Kortelainen, M. V. Gils, and J. Parkka, "Multichannel bed pressure 1124 sensor for sleep monitoring," presented at the Comput. Cardiol. Conf., 1125 Kraków, Poland, 2012.

[55] C. Brueser, A. Kerekes, S. Winter et al., "Multi-channel optical sensor- 1127 array for measuring ballistocardiograms and respiratory activity in bed," 1128 presented at the IEEE 34th Ann. Int. Conf. Eng. Med. Biol. Soc., San 1129 Diego, CA, USA, 2012.

[56] T. Watanabe and K. Watanabe, "Noncontact method for sleep stage esti- 1131 mation," IEEE Trans. Biomed. Eng., vol. 51, no. 10, pp. 1735-1748, Oct. 1132 2004.

[57] D. W. Jung, S. H. Hwang, H. N. Yoon et al., "Nocturnal awakening 1134 and sleep efficiency estimation using unobtrusively measured ballisto- 1135 cardiogram," IEEE Trans. Biomed. Eng., vol. 61, no. 1, pp. 131-138, Jan. 1136 2014.

[58] J. M. Kortelainen, M. O. Mendez, A. M. Bianchi et al., "Sleep staging 1138 based on signals acquired through bed sensor," IEEE Trans Inf. Technol. 1139 Biomed., vol. 14, no. 3, pp. 776-785, May 2010.

[59] M. Migliorini, A. M. Bianchi, Nistico et al., "Automatic sleep staging 1141 based on ballistocardiographic signals recorded through bed sensors," in 1142 Proc. IEEE Annu. Int. Conf. Eng. Med. Biol. Soc., 2010, pp. 3273-3276. 1143

[60] K. Tanida, M. Shibata, and M. M. Heitkemper, "Sleep stage assessment 1144 using power spectral indices of heart rate variability with a simple algo- 1145 rithm: limitations clarified from preliminary study," Biol.Res. Nursing, 1146 vol. 15, pp. 264-272, 2013.

[61] T. Koivistoinen, S. Junnila, A. Varri et al., "A new method for measuring 1148 the ballistocardiogram using EMFi sensors in a normal chair," in Proc. 1149 IEEE 26th Annu. Int. Conf. Eng. Med. Biol. Soc., 2004, pp. 2026-2029. 1150 
[62] M. Walter, B. Eilebrecht, T. Wartzek et al., "The smart car seat: Personalized monitoring of vital signs in automotive applications," Pers. Ubiquitous Comput., vol. 15, pp. 707-715, 2011.

[63] Y. Lim, K. Hong, K. Kim et al., "Monitoring physiological signals using nonintrusive sensors installed in daily life equipment," Biomed. Eng. Lett., vol. 1, pp. 11-20, 2011.

[64] B. H. Jansen, B. H. Larson, and K. Shankar, "Monitoring of the ballistocardiogram with the static charge sensitive bed," IEEE Trans. Biomed. Eng., vol. 38, no. 8, pp. 748-751, Aug. 1991.

[65] J. H. Shin, B. H. Choi, Y. G. Lim et al., "Automatic ballistocardiogram (BCG) beat detection using a template matching approach," in Proc. IEEE 30th Annu. Int. Conf. Eng. Med. Biol. Soc., 2008, pp. 1144-1146.

[66] D. Friedrich, X. L. Aubert, H. Fuhr et al., "Heart rate estimation on a beatto-beat basis via ballistocardiography - a hybrid approach," presented at the IEEE 32nd Annu. Int. Conf. Eng. Med. Biol. Soc., Buenos Aires, Argentina, 2010.

[67] S. Sprager and D. Zazula, "Optimization of heartbeat detection in fiberoptic unobtrusive measurements by using maximum a posteriori probability estimation," IEEE J. Biomed. Health Informat., vol. 18, no. 4, pp. 1161-1168, Jul. 2014.

[68] W. Xu, W. A. Sandham, A. C. Fisher et al., "Detection of the seismocardiogram W complex based on multiscale edges," presented at the 18th Аnnu. Int. Conf. IEEE EMBS (EMBC), Amsterdam, The Netherlands, 1996.

[69] C. Bruser, J. M. Kortelainen, S. Winter et al., "Improvement of forcesensor-based heart rate estimation using multi-channel data fusion," IEEE J. Biomed. Health Informat., 2014, to be published.

[70] J. Paalasmaa, H. Toivonen, and M. Partinen, "Adaptive heartbeat modelling for beat-to-beat heart rate measurement in ballistocardiograms," IEEE J. Biomed. Health Informat., 2014, to be published.

[71] C. Brueser, S. Winter, and S. Leonhardt, "Robust inter-beat interval estimation in cardiac vibration signals," Phys. Meas., vol. 34, pp. $123-$ 138,2013

[72] J. H. Shin, S. H. Hwang, M. H. Chang, et al., "Heart rate variability analysis using a ballistocardiogram during Valsalva manoeuvre and post exercise," Phys. Meas., vol. 32, pp. 1239-1264, 2011.

[73] X. Zhu, W. Chen, K. Kitamura et al., "Comparison of pulse rate variability indices estimated from pressure signal and photoplethysmogram," in Proc. IEEE Int. Conf. Biomed. Health Informat., 2012, pp. 867 -870.

[74] C. Brueser, S. Winter, and S. Leonhardt, "Unsupervised heart rate variability estimation from ballistocardiograms," presented at the 7th Int. Workshop Biosignal Interpretation, Como, Italy, 2012.

[75] O. T. Inan and G. T. A. Kovacs, "A low noise ac-bridge amplifier for ballistocardiogram measurement on an electronic weighing scale," Phys. Meas., vol. 31, pp. N51-N59, 2010.

[76] R. Wiard, O. Inan, B. Argyres et al., "Automatic detection of motion artifacts in the ballistocardiogram measured on a modified bathroom scale," Med. Biol. Eng. Comput., vol. 49, pp. 213-220, 2011.

[77] K. Pandia, S. Ravindran, R. Cole et al., "Motion artifact cancellation to obtain heart sounds from a single chest-worn accelerometer," presented at the IEEE Int. Conf. Acoust., Speech, Signal Process., Dallas, TX, USA, 2010.

[78] O. T. Inan, M. Etemadi, B. Widrow et al., "Adaptive cancellation of floor vibrations in standing ballistocardiogram measurements using a seismic sensor as a noise reference," IEEE Trans. Biomed. Eng., vol. 57, no. 3, pp. 722-727, Mar. 2010.

[79] I. Starr and S. Ogawa, "On the aging of the heart; why is it so much more conspicuous in the ballistocardiogram than in the pulse?" Amer. J. Med. Sci., vol. 242, pp. 399-410, 1961.

[80] O. T. Inan, G. T. A. Kovacs, and L. Giovangrandi, "Evaluating the lowerbody electromyogram signal acquired from the feet as a noise reference for standing ballistocardiogram measurements," IEEE Trans Inf. Technol. Biomed., vol. 14, no. 5, pp. 1188-1196, Sep. 2010.

[81] R. M. Wiard, O. T. Inan, L. Giovangrandi et al., "Preliminary results from standing ballistocardiography measurements in microgravity," in Proc. IEEE 35th Annu. Int. Conf. Eng. Med. Biol. Soc., 2013, pp. 7290-7293.

[82] I. Starr and A. Noordergraaf, Ballistocardiography in Cardiovascular Research: Physical Aspects of the Circulation in Health and Disease. Philadelphia, PA, USA: Lippincott, 1967.

[83] V. Gurev, K. Tavakolian, J. Constantino et al., "Mechanisms underlying isovolumic contraction and ejection peaks in seismocardiogram morphology," J. Med. Biol. Eng., vol. 32, pp. 103-110, 2012.

[84] Y. Henderson, "The mass movements of the circulation as shown by a recoil curve," Amer. J. Phys., vol. 14, pp. 287-298, 1905.
[85] W. R. Scarborough, S. A. Talbot, J. R. Braunstein et al., "Proposals 1226 for ballistocardiographic nomenclature and conventions: revised and ex- 1227 tended: Report of committee on ballistocardiographic terminology," Cir- 1228 culation, vol. 14, pp. 435-450, 1956.

[86] N. Westerhof, F. Bosman, C. J. De Vries et al., "Analog studies of the 1230 human systemic arterial tree," J. Biomech., vol. 2, pp. 121-134, 1969.1231

[87] J. Palladino, J. P. Mulier, F. Wu, et al., "Assessing in the state of the 1232 circulatory system via parameters versus variables," Cardiovasc. Diagn. 1233 Proc.., vol. 13, pp. 131-139, 1996.

[88] O. T. Inan, M. Etemadi, A. Paloma et al., "Non-invasive cardiac output 1235 trending during exercise recovery on a bathroom-scale-based ballistocar- 1236 diograph," Physiol. Meas., vol. 30, pp. 261-274, 2009.

[89] M. Etemadi, O. T. Inan, L. Giovangrandi et al., "Rapid assessment 1238 of cardiac contractility on a home bathroom scale," IEEE Trans. Inf. 1239 Technol. Biomed., vol. 15, no. 6, pp. 864-869, Nov. 2011.

[90] E. Pinheiro, O. Postolache, and P. Girao, "Pulse arrival time and bal- 1241 listocardiogram application to blood pressure variability estimation," in 1242 Proc. IEEE Int. Workshop Med. Meas. Appl., 2009, pp. 132-136. 1243

[91] R. Casanella, J. Gomez-Clapers, and R. Pallas-Areny, "On time interval 1244 measurements using BCG," in Proc. IEEE Annu. Int. Conf. Eng. Med. 1245 Biol. Soc., 2012, pp. 5034-5037.

[92] K. Tavakolian, G. Houlton, G. A. Durmont et al., "Precordial vibra- 1247 tions provide noninvasive detection of early-stage hemorrhage," J. Shock, 1248 vol. 41, pp. 91-96, 2014.

[93] M. Di Rienzo, E. Vaini, P. Castiglioni et al., "Beat-to-beat estimation of 1250 LVET and QS2 indices of cardiac mechanics from wearable seismocar- 1251 diography in ambulant subjects," in Proc. IEEE 35th Аnnu. Int. Conf. 1252 Eng. Med. Biol. Soc., 2013, pp. 7017-7020.

[94] S. M. Munir, R. C. Boganev, E. Sobash et al., "Devices in heart fail- 1254 ure: Potential methods for device-based monitoring of congestive heart 1255 failure," Texas Heart Inst. J., vol. 35, pp. 166-173, 2008.

[95] ClinicalTrials.gov. [Online]. Available: http://clinicaltrials.gov/show/ 1257 NCT01121107

[96] J. Zanetti and D. Salerno, "Seismocardiography: A new technique for 1259 recording cardiac vibrations. concept, method, and initial observations," 1260 J. Cardiovasc. Technol., vol. 9, pp. 111-120, 1990.

[97] A. M. Weissler, W. S. Harris, and C. D. Schoenfeld, "Systolic time 1262 intervals in heart failure in man," Circulation, vol. 37, pp. 149-159, 1263 1968.

[98] C. L. Garrard, A. M. Weissler, and H. T. Dodge, "The relationship of 1265 alterations in systolic time intervals to ejection fraction in patients with 1266 cardiac disease," Circulation, vol. 42, pp. 455-462, 1970.

[99] D. M. Salerno, J. M. Zanetti, L. A. Green et al., "Seismocardio- 1268 graphic changes associated with obstruction of coronary blood flow 1269 during balloon angioplasty;" Amer. J. Cardiol., vol. 68, pp. 201-207, 1270 1991.

100] D. Salerno, J. M. Zanetti, L. Poliac et al., "Exercise seismocardiography 1272 for detection of coronary artery disease," Amer. J. Noninvasive Cardiol., 1273 vol. 6, pp. 321-330, 1992.

[101] M. Jerosch-Herold, J. Zanetti, H. Merkle et al., "The seismocardiogram 1275 as magnetic-field-compatible alternative to the electrocardiogram for 1276 cardiac stress monitoring," Int. J. Cardiac Imag., vol. 15, pp. 523-531, 1277 1999.

[102] F. I. Marcus, V. Sorrell, J. Zanetti et al., "Accelerometer-derived time 1279 intervals during various pacing nodes in patients with biventricular pace- 1280 makers: Comparison with normals," Pacing Clin. Electrophysiol., vol. 1281 30, pp. 1476-1481, 2007.

[103] B. Silke, J. Spiers, N. Herity et al., "Seismocardiography during phar- 1283 macodynamic intervention in man," Automedia, vol. 16, pp. 35-44, 1284 1994.

[104] J. R. Libonati, J. Ciccolo, and J. Glassberg, "The tei index and exercise 1286 capacity," J. Sports Med., vol. 41, pp. 108-113, 2001.

[105] J. M. Zanetti and K. Tavakolian, "Seismocardiography: Past, present, and 1288 future," presented at the IEEE Eng. Med. Biol. Soc. Conf., Osaka, Japan, 1289 2013.

1290

[106] E. Pinheiro, O. Postolache, and P. Girao, "Theory and developments in 1291 an unobtrusive cardiovascular system representation: Ballistocardiogra- 1292 phy," Open Biomed. Eng. J., vol. 4, pp. 201-216, 2010.

[107] O. Postolache, P. S. Girao, J. Mendes et al., "Unobstrusive heart rate and 1294 respiratory rate monitor embedded on a wheelchair," in Proc. IEEE Int. 1295 Workshop Med. Meas. Appl., 2009, pp. 83-88.

[108] A. Akhbardeh, K. Tavakolian, V. Gurev et al., "Comparative analysis of 1297 three different modalities for characterization of the seismocardiogram," 1298 in Proc. IEEE Annu. Int. Conf. Eng. Med. Biol. Soc., 2009, pp. 2899- 1299 2903. 
1301 [109] C. Bruser, J. Diesel, M. D. H. Zink et al. "Automatic detection of 1302 1303

1304

1305

1306

1307

1308

1309

1310

1311

1312

1313 atrial fibrillation in cardiac vibration signals," IEEE J. Biomed. Health Informat., vol. 17, pp. 162-171, 2013.

[110] L. Giovangrandi, O. T. Inan, D. Banerjee et al., "Preliminary results from BCG and ECG measurements in the heart failure clinic," in Proc. IEEE Eng. Med. Biol. Soc. Annu. Int. Conf., 2012, pp. 3780-3783.

[111] A. I. Hernandez, F. Ziglio, A. Amblard et al., "Analysis of endocardial acceleration during intraoperative optimization of cardiac resynchronization therapy," in Proc. IEEE 35th Annu. Int. Conf. Eng. Med. Biol. Soc., 2013, pp. 7000-7003.

112] A. Scano and M. Strollo, "Ballistocardiographic research in weightlessness," Earth-Orient Appl. Space Technol., vol. 15, pp. 101-104, 1985.
[113] G. K. Prisk and P. F. Migeotte, "Physiological insights from gravity-free 1314 ballistocardiography," in Proc. IEEE 35th Annu. Int. Conf. Eng. Med. 1315 Biol. Soc., 2013, pp. 7282-7285.

[114] R. M. Baevsky, I. I. Funtova, E. S. Luchitskaya et al., "Microgravity: An 1317 ideal environment for cardiac force measurements," Cardiometry Open 1318 Access e-J., vol. 3, pp. 100-117, 2013. 
Q1. Author the abbreviation "EMFi" has been used for two terms, i.e., electromagnetic film and electromechanical film in the 1323 text. Please check.

Q2. Author: Please provide names of all authors in place of et al. in Refs. [1], [7], [8], [11]-[15], [17]-[19], [21], [22], [24]-[29], 1325 [32], [35]-[37], [42]-[45], [49], [51]-[53], [55], [57]-[59], [61]-[63], [65], [66], [68], [69], [72], [73], [76]-[78], [81], [83], 1326 [85]-[89], [92]-[94], [99]-[103], [107]-[111], and [114].

Q3. Author: Please provide the technical report number in Ref. [9].

Q4. Author: Please provide the page range in Ref. [23].

Q5. Author: Please update Refs. [69] and [70], if possible.

Q6. Author: Please provide the year in Ref. [95]. 